\title{
An RBF-FD method for pricing American options under jump-diffusion models
}

\author{
Majid Haghi $^{1}$, Reza Mollapourasl ${ }^{1,2}$, and Michèle Vanmaele ${ }^{3}$ \\ ${ }^{1}$ School of Mathematics, Shahid Rajaee Teacher Training University, Lavizan, Tehran 16788, Iran \\ ${ }^{2}$ Department of Mathematics, Oregon State University, Corvallis, OR 97331, USA \\ ${ }^{3}$ Department of Applied Mathematics, Computer Science and Statistics, Ghent University, 9000 Gent, Belgium \\ majidhaghi1363@gmail.com, mollapouresrttu.edu, michele.vanmaele@ugent.be
}

September 4, 2018

\begin{abstract}
American option prices under jump-diffusion models are determined as solutions to partial integro-differential equations (PIDE). In this paper a new combination of a time and spatial discretization applied to a linear complementary formulation (LCP) of the free boundary PIDE is proposed. First a coordinate stretching transformation is performed to the asset price so that the computation of the prices can be focused on regions of real interest instead of on the whole solution domain. An implicit-explicit time discretization applied to the reformulated LCP on a uniform temporal grid is followed by a spatial discretization to get a fully discrete system. The radial basis function $(\mathrm{RBF})$ finite difference method is a local method resulting in a sparse linear system in contrast to global RBF-methods which lead to ill-conditioned dense matrix systems. For the corresponding European option we prove consistency, stability and second-order convergence in a discrete $L_{2}$-norm. We derive mild conditions for the model parameters under which these results hold. Numerical experiments are performed with European and American options, and a comparison with numerical results available in the literature illustrates the accuracy and efficiency of the proposed algorithm.
\end{abstract}

Keywords: Radial basis functions, Finite difference, Option pricing, Merton's and Kou's models

\section{Introduction}

The pricing of European and American options under the jump-diffusion models introduced by Merton [30] and Kou [25] has been extensively studied in the literature aiming at faster and more accurate algorithms to solve the numerical approximating model. We will contribute to this literature by proposing an efficient numerical method which combines some existing techniques in an original way. The valuation of an option under a jump-diffusion model requires the solution to a partial integro-differential equation (PIDE). Before starting the discussion on the choice of the numerical method we first focus on the type of PIDE that is considered. In a first group of papers $[13,45,39,19,40,41,9,37]$ the solution to the PIDE is a function of the underlying price process $S$ itself. For the spatial discretization a uniform $S$-grid is used except in $[45,37]$ where a non-uniform grid is chosen to have a more refined grid near the strike. In a second group of papers [26, 27, 38, 8, 24, 22, 7, 23] the PIDE is transformed into a PIDE for the option price as a function of the log-returns. The differential operator has in that case constant coefficients. The spatial domain is further discretized by a uniform grid. Only in [7] a non-uniform grid is considered using the adaptive residual subsampling method from [14]. In our approach we consider a change of variable originally proposed by [10] and which is used in $[45,37]$ to select the non-uniform grid points. However, we will transform the PIDE to a PIDE for a function of this new variable $x$ resulting in a differential operator with coefficients depending on $x$ but having nice properties as we will show. In [34] a same transformation was applied but to a PDE problem that is solved using higher order finite differences while in [3] it is applied to a higher dimensional PDE. The advantage is that we can work with a uniform $x$-grid while the corresponding $S$-grid is refined around the point of irregularity of the payoff function. 
The American option pricing problem is a free boundary problem. One can also solve this free boundary problem itself under finite activity jump models by a so-called front-tracking method as in [7] or a front-fixing method as in [19]. The most common way is to formulate it as a linear complementary problem (LCP) involving a partial integro-differential inequality. In [37] the American option price formulated as a solution to an LCP is approximated by a Bermudian option price using a Richardson interpolation technique. In [12] a penalty method is proposed to approximate the resulting LCP. This penalty method is used in, e.g., [45, 40, 9] and is simple and efficient but only first order implying slow convergence. We will apply the operator splitting method as in, e.g., $[39,27,38,23]$ and introduced by [21] in the context of American option pricing. Its advantage is that no fixed point iteration techniques are needed at each time step in the discretized problem and that it has a a second-order convergence rate. Operator splitting is in fact a natural and old idea, namely decompose systems of P(I)DEs into simpler subproblems and treat those individually using specialized numerical algorithms.

For the spatial discretization of the PIDE typically a traditional finite difference method (FDM) is applied as in $[6,26,27,39,40]$ or a finite element method (FEM) as in $[20,29,49]$ for the PDE case or a finite volume method as in $[48,18]$. There are several other numerical methods available in the literature to solve the governing equation. For example in [1] an alternating direction implicit (ADI) finite difference method has been proposed. In $[33,35]$ the authors combined the spectral domain decomposition method and the Laplace transform method while in [9] a quadratic collocation method is considered. Recently radial basis functions (RBFs) methods have gained a lot of interest. In $[15,19,38,3,7]$ meshfree methods based on an RBF approximation have been shown to perform better than finite difference methods for option pricing problems in one or more spatial dimensions. However, the RBF collocation method in these papers is a global one and leads to a dense linear system which suffers from ill-conditioning. To overcome this drawback a local version of the method was proposed in order to have a sparse better-conditioned linear system. Local RBF-methods are applied in, e.g., [32, 31] for the pricing of American options with stochastic volatility and jump-diffusion model.

Over the last decade a relatively new method is developed in which the function derivatives are not approximated by derivatives of the RBF but by a linear combination of the function values of the RBF at some nodes. In [38] such method based on differential quadrature (DQ) is applied. However this RBF-DQ method leads to a full differentiation matrix since the RBFs are evaluated at all spatial discretization nodes. The radial basis function generated finite difference (RBF-FD) method is an FD method where the weights are computed by fitting an RBFinterpolant through some scattered nodes in one or more spatial dimensions resulting in a sparse differentiation matrix. Once the differentiation matrix is formed, it can be used repeatedly for spatial derivative approximation. Although the method has been implemented in various contexts in the last ten years, the first survey articles on RBF-FD are just now emerging, see, e.g., [16, 17]. In [24, 23] the local RBF-FD method is applied to price European and American options under jump-diffusion models when the $\mathrm{P}(\mathrm{I}) \mathrm{DE}$ for the option price is a function of the log-returns.

In order not to destroy the sparsity of the differentiation matrix by the non-local integral term originating from the jump part, we will combine the RBF-FD method with a three-level implicit-explicit (IMEX) time discretization where the integral term is treated explicitly. In [26, 27] an IMEX method with three time levels has been studied to evaluate the prices of European options under a jump diffusion model and the method is shown to be stable and second order accurate in a discrete $L_{2}$-norm. More recently, a class of IMEX-methods for pricing options under jump diffusion model has been proposed by [41]. We will consider the Crank-Nicolson-Leap-Frog scheme and show that the fully discrete scheme in our setting is also second order accurate when pricing European options. For American options we study the convergence numerically.

To summarize, the contribution of this paper is that we combine different techniques in an original way for the American option pricing under jump-diffusion models. We first perform in section 2 a coordinate stretching transformation resulting in a differential operator with non-constant coefficients in the PIDE of the reformulated LCP. Next an operator splitting method is applied in section 3. In section 4 an RBF-FD method is combined with a three-level implicit-explicit time discretization of section 3 that treats the non-local integral term explicitly. The fully discretized system has a sparse matrix and can be solved efficiently. Applying the differential matrices of section 4 to this solution an approximation for the hedging Greeks Delta and Gamma are obtained in section 6. In section 5 we carry out a stability and convergence analysis for the European option case dealing in particular with the non-constant coefficients in the differential operator. An extensive numerical study for European and American options and comparison with other schemes in the literature is carried out in section 7. 


\section{Option Pricing Model}

\subsection{Jump-diffusion model}

Consider a complete filtered probability space $\left(\Omega, \mathcal{F},(\mathcal{F})_{t \in[0, T]}, \mathbb{P}\right)$ where $T>0$ is a fixed finite time horizon. Let $\left(S_{t}\right)_{t \in[0, T]}$ be the price process of an asset which is modeled as a finite activity jump-diffusion, see e.g. [11]. Assume that there exists an equivalent martingale measure $\mathbb{Q}$ under which the dynamics of the asset price are given by the following stochastic differential equation (SDE) as in [41]

$$
\frac{\mathrm{d} S_{t}}{S_{t-}}=(r-q-\lambda \kappa) \mathrm{d} t+\sigma \mathrm{d} W_{t}+(Y-1) \mathrm{d} N_{t},
$$

where $W$ is a standard $\mathbb{Q}$-Brownian motion, $N$ is an independent Poisson process with intensity rate $\lambda>0, \kappa$ is the expected value of the random value $(Y-1)$ of the jump size distribution producing a jump from $S_{t-}$ to $y S_{t-}$ and with density $f(y)$ implying $\kappa=\int_{0}^{\infty}(y-1) f(y) \mathrm{d} y$. The parameters $r, q$ and $\sigma(>0)$, stand for the risk free interest rate, the continuous dividend yield and the volatility, respectively, and are for simplicity assumed to be constant. The case of time-dependent but deterministic functions requires a straightforward modification of the computations.

In this paper we focus on two popular jump-diffusion models with finite activity, Merton's [30] and Kou's [25] model. The jump size follows in the former a lognormal distribution and a double exponential one in the latter. Hence, the density function and corresponding mean are for $y>0$ respectively given by

$$
\begin{aligned}
\text { Merton: } f(y)=\frac{1}{y \gamma \sqrt{2 \pi}} \exp \left(-\frac{(\log (y)-\mu)^{2}}{2 \gamma^{2}}\right) \quad \kappa=\exp \left(\mu+\frac{\gamma^{2}}{2}\right)-1, \\
\text { Kou: } f(y)=p \alpha_{1} y^{-\alpha_{1}-1} \mathcal{H}(\log (y))+(1-p) \alpha_{2} y^{\alpha_{2}-1} \mathcal{H}(-\log (y)) \\
\kappa=p \frac{\alpha_{1}}{\alpha_{1}-1}+(1-p) \frac{\alpha_{2}}{\alpha_{2}+1}-1,
\end{aligned}
$$

with $\gamma>0, \mu \in \mathbb{R}, \alpha_{1}>1, \alpha_{2}>0$ and $0<p<1$ and where $\mathcal{H}(\cdot)$ is the Heaviside function with $\mathcal{H}(0)=1 / 2$.

\subsection{PIDE for European option}

By the Markov property, the fair value of a vanilla European (call or put) option at time $\tau$ is denoted $V(\tau, S)$ if the asset price at that time is $S_{\tau}=S$ with $\tau=T-t$ being the time to maturity and $T$ the expiration date of the contract. The following partial integro-differential equation (PIDE) can be derived for the evolution of $V(\tau, S)$ under the jump-diffusion models (1)-(3),

$$
\frac{\partial V}{\partial \tau}(\tau, S)=\mathcal{L} V(\tau, S), \quad \text { on }(0, T] \times(0, \infty)
$$

with

$$
\mathcal{L} V:=\frac{\sigma^{2} S^{2}}{2} \frac{\partial^{2} V}{\partial S^{2}}(\tau, S)+(r-q-\lambda \kappa) S \frac{\partial V}{\partial S}(\tau, S)-(r+\lambda) V(\tau, S)+\lambda \int_{0}^{\infty} V(\tau, S y) f(y) d y .
$$

The value at maturity is given as the initial condition

$$
V(0, S)=g(S), \text { for } S \in(0, \infty),
$$

where $g(S)$ is the payoff function of the claim, defined as

$$
g(S)= \begin{cases}\max \{K-S, 0\} & \text { put option, } \\ \max \{S-K, 0\} & \text { call option, }\end{cases}
$$

for a given strike price $K$. As boundary conditions for the European put option we impose

$$
V(\tau, 0)=K \mathrm{e}^{-r \tau}, \quad \lim _{S \rightarrow \infty} V(\tau, S)=0
$$

while for the call option we have

$$
V(\tau, 0)=0, \quad \lim _{S \rightarrow \infty}\left[V(\tau, S)-\left(S-K \mathrm{e}^{-r \tau}\right)\right]=0 .
$$

In what follows we focus on put options. The price of a call option will be computed through the put-call parity. 


\subsection{PIDE for American option}

The value of an American option which we also denote by $V(\tau, S)$ is a solution to the linear complementary problem (LCP) [42]

$$
\left\{\begin{array}{l}
\frac{\partial V}{\partial \tau}(\tau, S)-\mathcal{L} V(\tau, S) \geq 0 \\
V(\tau, S) \geq g(S), \\
\left(\frac{\partial V}{\partial \tau}(\tau, S)-\mathcal{L} V(\tau, S)\right)(V(\tau, S)-g(S))=0,
\end{array} \quad \text { for all }(\tau, S) \in(0, T] \times(0, \infty)\right.
$$

with the initial condition

$$
V(0, S)=g(S)
$$

where $g(S)$ is the payoff function. For American options we will focus on put options only. In that case, we impose in addition the following boundary conditions

$$
V(\tau, 0)=K, \quad \lim _{S \rightarrow \infty} V(\tau, S)=0 .
$$

\subsection{Localization, truncation and coordinate transformation}

To apply numerical techniques, we localize the variables and the integral term to bounded domains. First, we replace the unbounded domain $\Omega=[0, \infty)$ for $S$ with a bounded one $\tilde{\Omega}=\left[S_{\min }, S_{\max }\right]$. The values $S_{\min }$ and $S_{\max }$ will be chosen based on standard financial arguments with $K \in\left[S_{\min }, S_{\max }\right]$ and such that they are far enough away from the region of pricing interest in order for the solution to be unaffected by the truncation and for the asymptotic conditions to hold approximately. Accordingly, we truncate the integration domain in the integral term and divide it in two parts $\left[0, S_{\min } / S\right]$ and $\left[S_{\min } / S, S_{\max } / S\right]$. Utilizing the asymptotic behavior of the European put option, we approximate the integrand by $K \mathrm{e}^{-r \tau}-S y$ in the first integral while we change the variables in the second by putting $z=S y$, providing

$$
\lambda \int_{0}^{\infty} V(\tau, S y) f(y) d y \approx \lambda \int_{0}^{\frac{S_{\min }}{S}}\left(K \mathrm{e}^{-r \tau}-S y\right) f(y) d y+\frac{\lambda}{S} \int_{S_{\min }}^{S_{\max }} V(\tau, z) f\left(\frac{z}{S}\right) d z .
$$

The integral term for the call option case can be dealt with in a similar way.

The payoff function (6) and its derivatives are non-smooth at the strike $K$. Therefore, to reduce the loss of accuracy in the numerical approach we would like to have the points of the trial functions concentrated in a spatial region close to $S=K$. Hereto, we employ the following change of variable which transforms the physical domain $\tilde{\Omega}$ into the interval $[0,1]$,

$$
x(S)=\frac{\sinh ^{-1}(\zeta(S-K))-\sinh ^{-1}\left(\zeta\left(S_{\min }-K\right)\right)}{\sinh ^{-1}\left(\zeta\left(S_{\max }-K\right)\right)-\sinh ^{-1}\left(\zeta\left(S_{\min }-K\right)\right)} .
$$

The choice of the stretching parameter $\zeta$ determines the concentration near $S=K$. The inverse transformation is

$$
S(x)=\frac{1}{\zeta} \sinh \left(x \sinh ^{-1}\left(\zeta\left(S_{\max }-K\right)\right)+(1-x) \sinh ^{-1}\left(\zeta\left(S_{\min }-K\right)\right)\right)+K .
$$

This change of variable has originally been proposed in [10] and has been applied, for example in [4, 34, 32].

Finally, applying the chain rule we derive the following PIDE for $u(\tau, x)$ on the transformed truncated domain from the PIDE (4)-(5) for $V(\tau, S(x))$ on the original domain,

$$
\frac{\partial u}{\partial \tau}(\tau, x)=\mathcal{L} u(\tau, x) \quad \text { with } \mathcal{L} u(\tau, x)=\mathcal{D} u(\tau, x)+\mathcal{I} u(\tau, x)-\lambda u(\tau, x), \quad \text { on }(0, T] \times(0,1)
$$

where

$$
\mathcal{D} u(\tau, x):=\alpha(x) \frac{\partial^{2} u}{\partial x^{2}}(\tau, x)+\beta(x) \frac{\partial u}{\partial x}(\tau, x)-r u(\tau, x)
$$

with

$$
\alpha(x)=\frac{\sigma^{2}(S(x))^{2}}{2\left(S^{\prime}(x)\right)^{2}}, \quad \beta(x)=(r-q-\lambda \kappa) \frac{S(x)}{S^{\prime}(x)}-\alpha(x) \frac{S^{\prime \prime}(x)}{S^{\prime}(x)}
$$


and where

$$
\mathcal{I} u(\tau, x):=\lambda \int_{0}^{\frac{S_{\min }}{S(x)}}\left(K \mathrm{e}^{-r \tau}-S(x) y\right) f(y) d y+\lambda \int_{0}^{1} u(\tau, z) f\left(\frac{S(z)}{S(x)}\right) \frac{S^{\prime}(z)}{S(x)} d z .
$$

The corresponding boundary and initial conditions are

$$
\begin{gathered}
u(\tau, 0)=K \mathrm{e}^{-r \tau}-S_{\min }, \quad u(\tau, 1)=0, \quad \tau \in(0, T], \\
u(0, x)=g(S(x)), \quad x \in[0,1] .
\end{gathered}
$$

Along similar lines, the LCP (7) for American put options prices $V(\tau, S(x))$ is replaced by an LCP for $u(\tau, x)$ on the transformed truncated domain:

$$
\left\{\begin{array}{l}
u_{\tau}(\tau, x)-(\mathcal{D}-\lambda I) u(\tau, x)-\mathcal{I} u(\tau, x) \geq 0 \\
u(\tau, x) \geq g(S(x)) \\
\left(u_{\tau}(\tau, x)-(\mathcal{D}-\lambda I) u(\tau, x)-\mathcal{I} u(\tau, x)\right)(u(\tau, x)-g(S(x)))=0,
\end{array} \quad \text { on }(0, T] \times(0,1)\right.
$$

where $u_{\tau}$ stands for the partial derivative w.r.t. $\tau, I$ is an identity operator, $\mathcal{D}$ is defined by (10), and the integral operator $\mathcal{I}$ by

$$
\mathcal{I} u(\tau, x)=\lambda \int_{0}^{\frac{S_{\min }}{S(x)}}(K-S(x) y) f(y) d y+\lambda \int_{0}^{1} u(\tau, z) f\left(\frac{S(z)}{S(x)}\right) \frac{S^{\prime}(z)}{S(x)} d z .
$$

The corresponding boundary and initial conditions are

$$
\begin{gathered}
u(\tau, 0)=K, \quad u(\tau, 1)=0, \quad \tau \in(0, T], \\
u(0, x)=g(S(x)) \quad x \in[0,1] .
\end{gathered}
$$

\section{Time discretization with three time levels}

Let $\triangle \tau=\frac{T}{M}$ with integer $M \geq 1$ be a given time step and let the corresponding temporal grid points be given by $\tau_{k}=k \triangle \tau$ for $0 \leq k \leq M$. We apply an implicit-explicit (IMEX) time semi-discretization with three time levels to the PIDE (9). In particular, we consider the Crank-Nicolson-Leap-Frog (CNLF) scheme as in [2] and [24]. In [41] this type of scheme is called the IMEX-midpoint scheme. The differential part is treated implicitly, while the integral part is treated explicitly. In order to start the algorithm we will need initial data for $k=0$ and the value for $k=1$ is obtained by an implicit-explicit backward difference method of order one (IMEX-BDF1). Thus, denoting $U^{k}(x):=u\left(\tau_{k}, x\right)$, the PIDE (9) for the price of a European option is approximated by following implicit-explicit time semi-discrete scheme for $x \in(0,1)$

$$
\begin{gathered}
\frac{U^{1}(x)-U^{0}(x)}{\triangle \tau}=(\mathcal{D}-\lambda I) U^{1}(x)+\mathcal{I} U^{0}(x), \\
\frac{U^{k+1}(x)-U^{k-1}(x)}{2 \triangle \tau}=(\mathcal{D}-\lambda I) \frac{U^{k+1}(x)+U^{k-1}(x)}{2}+\mathcal{I} U^{k}(x), \quad \text { for } \quad 1 \leq k \leq M-1,
\end{gathered}
$$

with boundary and initial conditions

$$
U^{k}(0)=K \mathrm{e}^{-r \tau}-S_{\min }, \quad U^{k}(1)=0, \text { for } 1 \leq k \leq M \quad \text { and } \quad U^{0}(x)=g(S(x)) \text { for } x \in[0,1] .
$$

For American options, we use the operator splitting method for solving the time semi-discretization of the LCP problem (15). This method which was introduced by Ikonen and Toivanen in [21] to evaluate the price of the American put option under the Black-Scholes model, and which was studied by Toivanen in [45] under the Kou model, starts from the reformulated LCP (15) in terms of an auxiliary function $\Lambda(\tau, x)$,

$$
\left\{\begin{array}{l}
u_{\tau}(\tau, x)-(\mathcal{D}-\lambda I) u(\tau, x)-\mathcal{I} u(\tau, x)=\Lambda(\tau, x), \Lambda(\tau, x) \geq 0 \\
u(\tau, x) \geq g(S(x)) \\
\Lambda(\tau, x)(u(\tau, x)-g(S(x)))=0
\end{array} \quad \text { on }(0, T] \times(0,1),\right.
$$


with boundary and initial conditions (17)-(18).

Then the operator splitting method splits the first equation on each time level into two discrete equations using some intermediate solution $\widetilde{U}^{k+1}(x)$. Let $\Lambda^{k}(x):=\Lambda\left(\tau_{k}, x\right)$. For the CNLF scheme we find for $x \in(0,1)$ and similarly to [27]

$$
\left\{\begin{array}{l}
\frac{\widetilde{U}^{1}(x)-U^{0}(x)}{\triangle \tau}-\left((\mathcal{D}-\lambda I) \widetilde{U}^{1}(x)+\mathcal{I} U^{0}(x)\right)=\Lambda^{1}(x), \\
\Lambda^{1}(x)=\Lambda^{0}(x)+\frac{U^{1}(x)-\widetilde{U}^{1}(x)}{\triangle \tau}, \\
\Lambda^{1}(x) \geq 0, U^{1}(x) \geq g(S(x)), \Lambda^{1}(x)\left(U^{1}(x)-g(S(x))\right)=0,
\end{array}\right.
$$

and

$$
\begin{cases}\frac{\widetilde{U}^{k+1}(x)-U^{k-1}(x)}{2 \triangle \tau}-\left((\mathcal{D}-\lambda I) \frac{\widetilde{U}^{k+1}(x)+U^{k-1}(x)}{2}+\mathcal{I} U^{k}(x)\right)=\Lambda^{k+1}(x), & \\ \Lambda^{k+1}(x)=\Lambda^{k}(x)+\frac{U^{k+1}(x)-\widetilde{U}^{k+1}(x)}{2 \triangle \tau}, & 1 \leq k \leq M-1 . \\ \Lambda^{k+1}(x) \geq 0, U^{k+1}(x) \geq g(S(x)), \quad \Lambda^{k+1}(x)\left(U^{k+1}(x)-g(S(x))\right)=0, & \end{cases}
$$

with boundary and initial conditions

$$
U^{k}(0)=K, \quad U^{k}(1)=0, \text { for } 1 \leq k \leq M \quad \text { and } \quad U^{0}(x)=g(S(x)) \text { for } x \in[0,1] .
$$

\section{Spatial discretization}

The time semi-discrete systems in previous section are further discretized in space to get fully discretized systems of equations that can be easily computed numerically. We will apply the local radial basis function method which was independently introduced by several authors $[43,46,47]$. This local RBF method can be viewed as a generalization of the classical finite difference (FD) method, and is therefore also called RBF-FD method. In the FD method the weights are computed using polynomial interpolation while in the RBF-FD method they are computed by fitting an RBF interpolant through a grid point and some neighbor points. In this sense it is a local RBF method in contrast to a global RBF method where all grid points in the spatial domain are taken into consideration to determine the interpolation coefficients resulting in a dense linear system showing ill-conditioning. The resulting linear system in the local RBF method is sparse, hence overcoming the ill-conditioning of the global method.

\subsection{RBF-FD based approximation}

Consider a spatial domain $\Omega \subset \mathbb{R}^{d}$ and a set of distinct RBF collocation points $\mathbf{X}=\left\{\mathbf{x}_{1}, \mathbf{x}_{2}, \ldots, \mathbf{x}_{N}\right\}$ in $\Omega$. Let $\mathbf{X}_{j}=\left\{\mathbf{x}_{1}^{(j)}, \ldots, \mathbf{x}_{n}^{(j)}\right\} \subset \mathbf{X}$ be a subset containing $\mathbf{x}_{j}$ and its $n-1$ nearest neighboring points forming a stencil with $\mathbf{x}_{j}$ as center and $n \ll N$. The number of points $n$ in each stencil can be either constant or vary with $j$.

In the RBF-FD approach any linear differential operator as for example $\mathcal{D}$ defined by (10) acting on $u(\mathbf{x})$ evaluated at $\mathbf{x}_{j}$, is approximated by a linear weighted combination of the function values of $u$ at the points of $\mathbf{X}_{j}$,

$$
\mathcal{D} u\left(\mathbf{x}_{j}\right) \approx \sum_{k=1}^{n} w_{k}^{(j)} u\left(\mathbf{x}_{k}^{(j)}\right) .
$$

The RBF-FD weights, $w_{k}^{(j)}, k=1, \ldots, n$, are found by enforcing that the approximation is exact within the space spanned by the RBFs $\left\{\phi\left(\left\|\mathbf{x}-\mathbf{x}_{i}^{(j)}\right\|\right)\right\}_{i=1}^{n}$, centered at the nodes $\mathbf{x}_{i}^{(j)}, i=1, \ldots, n$, and with $\|\cdot\|$ the standard Euclidean 2-norm, so that

$$
\mathcal{D} \phi\left(\left\|\mathbf{x}_{j}-\mathbf{x}_{i}^{(j)}\right\|\right)=\sum_{k=1}^{n} w_{k}^{(j)} \phi\left(\left\|\mathbf{x}_{i}^{(j)}-\mathbf{x}_{k}^{(j)}\right\|\right), \quad i=1, \ldots, n .
$$

This is an $n \times n$ linear algebraic system

$$
\mathbf{A}^{(j)} \mathbf{W}^{(j)}=\mathbf{C}^{(j)}
$$


where the coefficient matrix $\mathbf{A}^{(j)}$ has entries $a_{i k}^{(j)}=\phi\left(\left\|\mathbf{x}_{i}^{(j)}-\mathbf{x}_{k}^{(j)}\right\|\right)$, for $i, k=1, \ldots, n, \mathbf{W}^{(j)}$ is the differential weights vector, and the right hand side is defined by $c_{i}^{(j)}=\mathcal{D} \phi\left(\left\|\mathbf{x}_{j}-\mathbf{x}_{i}^{(j)}\right\|\right), i=1, \ldots, n$. We have to solve this $n \times n$ linear system for each stencil center $\mathbf{x}_{j}, j=1, \ldots, N$ to form the $N$ rows of the sparse differentiation matrix with $n$ non-zeros per row. In the context of time-dependent P(I)DEs, the stencil weights remain constant for all time-steps when the nodes are stationary.

Table 1 lists some radial basis functions that are commonly employed in the literature. Some of them depend on a shape parameter $\epsilon$ that should be chosen in an optimal way to minimize the approximation error.

\begin{tabular}{lc}
\hline Function name & Definition \\
\hline Gaussian (GA) & $\exp \left(-\epsilon^{2} r^{2}\right)$ \\
Multiquadrics (MQ) & $\sqrt{1+(\epsilon r)^{2}}$ \\
Inverse multiquadrics (IMQ) & $\frac{1}{\sqrt{1+(\epsilon r)^{2}}}$ \\
Conical splines & $r^{2 k+1}$ \\
Thin plate splines (TPS) & $(-1)^{k+1} r^{2 k} \log (r)$ \\
\hline
\end{tabular}

Table 1: Some well-known radial basis functions

Obviously, since $n \ll N$ the size of the linear systems (23) is much smaller than the size $N \times N$ of the linear system of a global RBF collocation method. A global RBF method to derive a differentiation matrix needs $O\left(N^{3}\right)$ operations, and results in a dense matrix. In the RBF-FD method we only need $O\left(n^{3}\right)$ operations for each of the $N$ stencils, so that the total cost of computing is $O\left(n^{3} N\right)$, without taking into account the cost of determining the stencil grids. For $n$ fixed with $n \ll N$, the total cost will be $O(N)$ for increasing $N$. The weights can be computed by inverting the local distance matrices $\mathbf{A}^{(j)}$ of order $n \times n$ for each stencil. These distance matrices depend only on the distance of the grid points implying that for uniform grids we only need to compute the inverse of one local distance matrix. Further, the differentiation matrix for one stencil is independent from those for the other stencils. Hence their computation can be parallellized to increase the efficiency of RBF-FD method in high dimensional problems and adaptive algorithms.

\subsection{Discretization of the integral operator}

Besides the linear differential operator $\mathcal{D}$ we also have to discretize in space the integral operator $\mathcal{I}$ defined in (12) and (16) for a European and an American put option respectively. First, note that the expressions (12) and (16) only differ by the factor $\mathrm{e}^{-r \tau}$ in the first term. Moreover, this first term does not depend on the unknown function $u$ and can be evaluated exactly for the density functions $f(\cdot)(2)-(3)$ of the Merton and the Kou model. Apart from the factor $\lambda$, introducing the indicator functions $1_{\text {European }}$ and $1_{\text {American }}$, we find for the first integral term

$$
\mathcal{R}(\tau, x):=\int_{0}^{\frac{S_{\min }}{S(x)}}\left(K\left(1_{\text {European }} \mathrm{e}^{-r \tau}+1_{\text {American }}\right)-S(x) y\right) f(y) d y,
$$

where for the Merton model using the cumulative distribution function $\Phi(\cdot)$ of a standard normal random variable

$$
\mathcal{R}(\tau, x)=K\left(1_{\text {European }} \mathrm{e}^{-r \tau}+1_{\text {American }}\right) \Phi\left(\frac{\log \left(\frac{S_{\min }}{S(x)}\right)-\mu}{\gamma}\right)-S(x) \mathrm{e}^{\mu+\frac{1}{2} \gamma^{2}} \Phi\left(\frac{\log \left(\frac{S_{\min }}{S(x)}\right)-\mu-\gamma^{2}}{\gamma}\right),
$$

and for the Kou model recalling that $S_{\min } / S(x)<1$

$$
\mathcal{R}(\tau, x)=(1-p)\left(\frac{S_{\min }}{S(x)}\right)^{\alpha_{2}}\left(K\left(1_{\text {European }} \mathrm{e}^{-r \tau}+1_{\text {American }}\right)-\frac{\alpha_{2}}{\alpha_{2}+1} S_{\min }\right) .
$$

The second integral in (12) and (16) is approximated by the trapezoidal rule

$$
\lambda \int_{0}^{1} u(\tau, z) f\left(\frac{S(z)}{S(x)}\right) \frac{S^{\prime}(z)}{S(x)} d z \approx \frac{\lambda \triangle z}{2}\left(\sum_{j=0}^{N} \omega_{j} F_{j}(x) u\left(\tau, z_{j}\right)\right)
$$

where $z_{j}=j \triangle z$ with $\triangle z=\frac{1}{N}, \omega_{j}=1$ for $j=0, N$ and $\omega_{j}=2$ for $j=1, \ldots, N-1$, and $F_{j}(x)=$ $f\left(\frac{S\left(z_{j}\right)}{S(x)}\right) \frac{S^{\prime}\left(z_{j}\right)}{S(x)}$. 


\subsection{Fully discretized system for European option}

We now apply the RBF-FD approximation of section 4.1 with $d=1$. Let $X=\left\{x_{0}, x_{2}, \ldots, x_{N}\right\}$ be a set of distinct interpolation points of $[0,1]$ with $x_{0}=0$ and $x_{N}=1$, and $\mathcal{T}=\left\{\tau_{0}=0<\tau_{1}<\cdots<\tau_{M}=T\right\}$ be a partition of $[0, T]$. Applying the RBF-FD method in one dimension and the discretization of the integral operator as described in the previous subsections to the time semi-discrete problem (19)-(20), the evaluation of a European put option price is reduced to finding an approximation $\mathbf{U}^{k}:=\left[\begin{array}{llll}U_{0}^{k} & U_{1}^{k} & \cdots & U_{N}^{k}\end{array}\right]^{\top}$ for $\left[u\left(\tau_{k}, x_{0}\right) \quad u\left(\tau_{k}, x_{1}\right) \quad \cdots u\left(\tau_{k}, x_{N}\right)\right]^{\top}, k=0, \ldots, M$, as a solution to the following time stepping scheme,

$$
\begin{gathered}
\frac{\mathbf{U}^{1}-\mathbf{U}^{0}}{\Delta \tau}=(\mathcal{D}-\lambda \mathbf{I}) \mathbf{U}^{1}+\mathcal{I} \mathbf{U}^{0}, \quad \text { with } \mathbf{U}^{0}=\mathbf{g}, \\
\frac{\mathbf{U}^{k+1}-\mathbf{U}^{k-1}}{2 \Delta \tau}=(\mathcal{D}-\lambda \mathbf{I})\left(\frac{\mathbf{U}^{k+1}+\mathbf{U}^{k-1}}{2}\right)+\mathcal{I} \mathbf{U}^{k}, \quad \text { for } 1 \leq k \leq M-1,
\end{gathered}
$$

where $\mathcal{D}$ is the differentiation matrix associated with the differential operator $\mathcal{D}(10)$, $\mathbf{I}$ is an identity matrix of order $N+1$ and $\mathcal{I}$ is the integral matrix corresponding to the integral operator $\mathcal{I}$ (12) which can be expressed as $\mathcal{I} \mathbf{U}^{k}=\lambda \mathcal{J} \mathbf{U}^{k}+\lambda \mathcal{R}^{k}$ with according to (27) $\mathcal{J}=\left(\frac{\triangle z}{2} \omega_{j} F_{j}\left(x_{i}\right)\right)_{0 \leq i, j \leq N}$ and according to (24) $\mathcal{R}^{k}:=\left[\begin{array}{llll}\mathcal{R}\left(\tau_{k}, x_{0}\right) & \mathcal{R}\left(\tau_{k}, x_{1}\right) & \ldots & \mathcal{R}\left(\tau_{k}, x_{N}\right)\end{array}\right]^{\top}$. In the boundary points $x_{0}=0$ and $x_{N}=1$ the boundary conditions $U_{0}^{k}=u\left(\tau_{k}, 0\right)=K \mathrm{e}^{-r \tau_{k}}-S_{\min }, U_{N}^{k}=u\left(\tau_{k}, 1\right)=0$ are imposed according to (13). Note that it is possible to enforce the boundary conditions in (28)-(29) by putting the elements zero in the first and last row of the matrices on the right hand side. The initial vector $\mathbf{U}^{0}$ is the given vector $\mathbf{g}$ defined by $\left[\begin{array}{llll}g\left(S\left(x_{0}\right)\right) & g\left(S\left(x_{1}\right)\right) & \cdots & g\left(S\left(x_{N}\right)\right)\end{array}\right]^{\top}$ where $g(\cdot)$ is the payoff function (6). Then, the vector $\mathbf{U}^{M}$ provides an approximation to the European put option price at time zero for different initial asset prices $S\left(x_{j}\right)$, $j=0, \ldots, N$.

\subsection{Fully discretized system for American option}

As for the European put option, we apply the RBF-FD method using a set of $N+1$ collocation points $X=$ $\left\{x_{0}, x_{1}, \ldots, x_{N}\right\}$ of the interval $[0,1]$ with $x_{0}=0$ and $x_{N}=1$ and the discretization of the integral operator to the time semi-discrete systems (21)-(22) of the LCP problem with operator splitting. To evaluate an American put option price, we look for an approximation $\mathbf{U}^{k}:=\left[\begin{array}{lllll}U_{0}^{k} & U_{1}^{k} & \cdots & U_{N}^{k}\end{array}\right]^{\top}$ for $\left[\begin{array}{lllll}u\left(\tau_{k}, x_{0}\right) & u\left(\tau_{k}, x_{1}\right) & \cdots & u\left(\tau_{k}, x_{N}\right)\end{array}\right]^{\top}$, $k=0, \ldots, M$, as a solution to the time stepping schemes

$$
\left\{\begin{array}{l}
\frac{\widetilde{\mathbf{U}}^{1}-\mathbf{U}^{0}}{\Delta \tau}-\left((\mathcal{D}-\lambda \mathbf{I}) \widetilde{\mathbf{U}}^{1}+\mathcal{I} \mathbf{U}^{0}\right)=\boldsymbol{\Lambda}^{0}, \quad \mathbf{U}^{0}=\mathbf{g} \\
\boldsymbol{\Lambda}^{1}=\boldsymbol{\Lambda}^{0}+\frac{\mathbf{U}^{1}-\widetilde{\mathbf{U}}^{1}}{\Delta \tau} \\
\boldsymbol{\Lambda}^{1} \geq \mathbf{0}, \quad \mathbf{U}^{1} \geq \mathbf{g}, \quad\left(\boldsymbol{\Lambda}^{1}\right)^{\top}\left(\mathbf{U}^{1}-\mathbf{g}\right)=0
\end{array}\right.
$$

and

$$
\begin{cases}\frac{\widetilde{\mathbf{U}}^{k+1}-\mathbf{U}^{k-1}}{2 \triangle \tau}-\left((\mathcal{D}-\lambda \mathbf{I}) \frac{\widetilde{\mathbf{U}}^{k+1}+\mathbf{U}^{k-1}}{2}+\mathcal{I} \mathbf{U}^{k}\right)=\boldsymbol{\Lambda}^{k} & \\ \boldsymbol{\Lambda}^{k+1}=\boldsymbol{\Lambda}^{k}+\frac{\mathbf{U}^{k+1}-\widetilde{\mathbf{U}}^{k+1}}{2 \triangle \tau}, & 1 \leq k \leq M-1 \\ \boldsymbol{\Lambda}^{k+1} \geq \mathbf{0}, \quad \mathbf{U}^{k+1} \geq \mathbf{g}, \quad\left(\boldsymbol{\Lambda}^{k+1}\right)^{\top}\left(\mathbf{U}^{k+1}-\mathbf{g}\right)=0, & \end{cases}
$$

where $\widetilde{\mathbf{U}}^{k+1}$ is an intermediate solution vector, $\Lambda^{k}:=\left[\begin{array}{llll}\Lambda\left(\tau_{k}, x_{0}\right) & \Lambda\left(\tau_{k}, x_{1}\right) & \cdots & \Lambda\left(\tau_{k}, x_{N}\right)\end{array}\right]^{\top}$ is the auxiliary function $\Lambda(\tau, x)$ evaluated at the discretization points and $\mathbf{g}$ stands for the vector $\left[\begin{array}{llll}g\left(S\left(x_{0}\right)\right) & g\left(S\left(x_{1}\right)\right) & \cdots & g\left(S\left(x_{N}\right)\right)\end{array}\right]^{\top}$ with $g(\cdot)$ the payoff function (6). $\mathcal{D}$ is the differentiation matrix associated with the differential operator $\mathcal{D}(10)$, I is an identity matrix of order $N+1$ and $\mathcal{I}$ is the integral matrix corresponding to the integral operator $\mathcal{I}$ (16) which can be expressed as $\mathcal{I} \mathbf{U}^{k}=\lambda \mathcal{J} \mathbf{U}^{k}+\lambda \mathcal{R}^{k}$ with according to (27) $\mathcal{J}=\left(\frac{\triangle z}{2} \omega_{j} F_{j}\left(x_{i}\right)\right)_{0 \leq i, j \leq N}$ and according to (24) $\mathcal{R}:=\left[\begin{array}{llll}\mathcal{R}\left(\tau_{k}, x_{0}\right) & \mathcal{R}\left(\tau_{k}, x_{1}\right) & \cdots & \mathcal{R}\left(\tau_{k}, x_{N}\right)\end{array}\right]^{\top}$ which 
does not depend on $\tau_{k}$. In the boundary points $x_{0}=0$ and $x_{N}=1$ the boundary conditions $U_{0}^{k}=u\left(\tau_{k}, 0\right)=K$, $U_{N}^{k}=u\left(\tau_{k}, 1\right)=0$ are imposed according to (17).

Each time step is split into two parts. Starting from the initial vector $\mathbf{U}^{0}=\mathbf{g}$ and $\boldsymbol{\Lambda}^{0}=\mathbf{0}$, first, the intermediate solution vector $\widetilde{\mathbf{U}}^{1}$ is solved from the system of linear equations (30)(a). Next, to compute $\Lambda^{1}$ and $\mathbf{U}^{1}$ from (b) satisfying relations (c) we use the equivalent relations $\mathbf{U}^{1}=\max \left(\mathbf{g}, \widetilde{\mathbf{U}}^{1}-\triangle \tau \boldsymbol{\Lambda}^{0}\right)$ and $\boldsymbol{\Lambda}^{1}=\boldsymbol{\Lambda}^{0}+\frac{\mathbf{U}^{1}-\widetilde{\mathbf{U}}^{1}}{\Delta \tau}$. Now, we can use the system (31) for $k=1, \ldots, M-1$. For the computed values $\mathbf{U}^{k-1}, \mathbf{U}^{k}$ and $\Lambda^{k}$, the intermediate solution vector $\widetilde{\mathbf{U}}^{k+1}$ is first solved from the modified system of linear equations (31)(a). Next, the update step (b) satisfying (c) can be performed very fast and at each spatial grid point independently with the formulas $\mathbf{U}^{k+1}=$ $\max \left(\mathbf{g}, \widetilde{\mathbf{U}}^{k+1}-2 \triangle \tau \boldsymbol{\Lambda}^{k}\right)$ and $\boldsymbol{\Lambda}^{k+1}=\boldsymbol{\Lambda}^{k}+\frac{\mathbf{U}^{k+1}-\widetilde{\mathbf{U}}^{k+1}}{2 \triangle \tau}$. Finally, we obtain $\mathbf{U}^{M}$ as an approximate value for the American put option price at time zero for different initial asset prices $S\left(x_{j}\right), j=0, \ldots, N$.

\section{Stability and convergence analysis}

In this section we analyze the stability and convergence of the fully discrete scheme (28)-(29) to price a European option in the case of a uniform grid in space and $n=3$ points per stencil. Further, we choose as RBF the multiquadratic function

$$
\phi(|x-y|)=\sqrt{\epsilon^{2}+|x-y|^{2}}
$$

where $\epsilon$ is the shape parameter. For the collocation points we focus on the interior points $x_{1}, \ldots, x_{N-1}$. We first construct the differential matrix $\mathbf{D}$ corresponding to the differential operator $\mathcal{D}(10)$ in this particular case and derive some properties. This differential matrix $\mathbf{D}$ is an $(N-1) \times(N-1)$-submatrix of the matrix $\mathcal{D}$ corresponding to the interior points obtained by deleting the first and last row and columns in $\mathcal{D}$. The grid points in the $x$-direction are equidistant with step size $h$ and each stencil centered around $x_{j}$ contains also the interpolation points $x_{j}-h$ and $x_{j}+h$. We further consider the grid points to be stationary such that the stencil weights remain constant for all time-steps. The first order derivative of $u\left(\tau_{k}, x\right)$ at $x=x_{j}$ for $j=1, \ldots, N-1$ and $k=0, \ldots, M$, is approximated by

$$
\frac{\partial u}{\partial x}\left(\tau_{k}, x_{j}\right) \approx a_{j-1} u\left(\tau_{k}, x_{j}-h\right)+a_{j} u\left(\tau_{k}, x_{j}\right)+a_{j+1} u\left(\tau_{k}, x_{j}+h\right)
$$

Requiring that for the multiquadratic RBF function (32) it holds that

$$
\frac{\partial \phi}{\partial x}\left(\left|x_{j}-x^{(j)}\right|\right)=a_{j-1} \phi\left(\left|x_{j}-h-x^{(j)}\right|\right)+a_{j} \phi\left(\left|x_{j}-x^{(j)}\right|\right)+a_{j+1} \phi\left(\left|x_{j}+h-x^{(j)}\right|\right),
$$

for $x^{(j)}=x_{j}-h, x^{(j)}=x_{j}$ and $x^{(j)}=x_{j}+h$, we get a linear algebraic system in three unknowns $a_{j-1}, a_{j}$, $a_{j+1}$ with solution, see [5],

$$
a_{j-1}=-a_{j+1}=-\frac{1}{4 h} \frac{1+\sqrt{1+\frac{4 h^{2}}{\epsilon^{2}}}}{\sqrt{1+\frac{h^{2}}{\epsilon^{2}}}}, \quad a_{j}=0
$$

When $\epsilon \gg h$, we obtain by Taylor's expansion and neglecting higher order terms, as in [5], that

$$
a_{j-1}=-a_{j+1} \approx-\frac{1}{2 h}\left(1+\frac{h^{2}}{2 \epsilon^{2}}\right), \quad a_{j}=0 .
$$

Note that these coefficients are independent of $j$ due to the equidistant grid points and only one linear system has to be solved. In what follows we denote

$$
a:=\frac{1}{2 h}\left(1+\frac{h^{2}}{2 \epsilon^{2}}\right)
$$

In a similar way we approximate the second order derivative of $u\left(\tau_{k}, x\right)$ at $x=x_{j}$ for $j=1, \ldots, N-1$ and $k=0, \ldots, M$, by

$$
\frac{\partial^{2} u}{\partial x^{2}}\left(\tau_{k}, x_{j}\right) \approx b_{j-1} u\left(\tau_{k}, x_{j}-h\right)+b_{j} u\left(\tau_{k}, x_{j}\right)+b_{j+1} u\left(\tau_{k}, x_{j}+h\right)
$$


where the unknown coefficients $b_{j-1}, b_{j}$ and $b_{j+1}$ are solved from a linear system consisting of the equations

$$
\frac{\partial^{2} \phi}{\partial x^{2}}\left(\left|x_{j}-x^{(j)}\right|\right)=b_{j-1} \phi\left(\left|x_{j}-h-x^{(j)}\right|\right)+b_{j} \phi\left(\left|x_{j}-x^{(j)}\right|\right)+b_{j+1} \phi\left(\left|x_{j}+h-x^{(j)}\right|\right),
$$

for $x^{(j)}=x_{j}-h, x^{(j)}=x_{j}$ and $x^{(j)}=x_{j}+h$. The solution is given by, see [5],

$$
b_{j-1}=b_{j+1}=\frac{2+\left(\frac{h^{2}}{\epsilon^{2}}+2\right) \sqrt{1+\frac{4 h^{2}}{\epsilon^{2}}}+\frac{5 h^{2}}{\epsilon^{2}}+\frac{2 h^{4}}{\epsilon^{4}}}{4 h^{2}\left(1+\frac{h^{2}}{\epsilon^{2}}\right)^{3 / 2}}, \quad b_{j}=-\frac{2+\left(\frac{h^{2}}{\epsilon^{2}}+2\right) \sqrt{1+\frac{4 h^{2}}{\epsilon^{2}}}+\frac{3 h^{2}}{\epsilon^{2}}}{2 h^{2}\left(1+\frac{h^{2}}{\epsilon^{2}}\right)} .
$$

When $\epsilon \gg h$, we can approximate these coefficients which are independent of $j$ respectively by, see again [5],

$$
\hat{b}:=\frac{1}{h^{2}}\left(1+\frac{h^{2}}{\epsilon^{2}}\right)(>0), \quad b:=-\frac{2}{h^{2}}\left(1+\frac{h^{2}}{\epsilon^{2}}\right)=-2 \hat{b}(<0) .
$$

Assuming that $\epsilon \gg h$, substitution of the first and second order derivative approximations (33) and (36) with coefficients (34)-(35) and (37) respectively in the expression (10) for the differential operator $\mathcal{D}$ leads to the tridiagonal differential matrix

$$
\mathbf{D}=\alpha(\mathbf{x}) \mathbf{D}_{\mathbf{x x}}+\beta(\mathbf{x}) \mathbf{D}_{\mathbf{x}}-r \mathbf{I},
$$

where

$$
\mathbf{D}_{\mathbf{x x}}=\left(\begin{array}{ccccc}
b & \hat{b} & & & \\
\hat{b} & b & \hat{b} & & \\
& \ddots & \ddots & \ddots & \\
& & \hat{b} & b & \hat{b} \\
& & & \hat{b} & b
\end{array}\right) \quad \text { and } \quad \mathbf{D}_{\mathbf{x}}=\left(\begin{array}{ccccc}
0 & a & & & \\
-a & 0 & a & & \\
& \ddots & \ddots & \ddots & \\
& & -a & 0 & a \\
& & & -a & 0
\end{array}\right)
$$

and where $\alpha(\mathbf{x})$ and $\beta(\mathbf{x})$ are diagonal matrices defined by

$$
\alpha(\mathbf{x})=\operatorname{diag}\left(\alpha\left(x_{1}\right), \alpha\left(x_{2}\right), \ldots, \alpha\left(x_{N-1}\right)\right), \quad \beta(\mathbf{x})=\operatorname{diag}\left(\beta\left(x_{1}\right), \beta\left(x_{2}\right), \ldots, \beta\left(x_{N-1}\right)\right)
$$

with $\alpha(x)$ and $\beta(x)$ given in (11). Hence, the non-zero elements of the differentiation matrix are

$$
\begin{gathered}
\mathbf{D}_{i i}=\alpha\left(x_{i}\right) b-r, \quad i=1,2, \ldots, N-1 \\
\mathbf{D}_{i+1, i}=\alpha\left(x_{i+1}\right) \hat{b}-\beta\left(x_{i+1}\right) a, \quad \mathbf{D}_{i, i+1}=\alpha\left(x_{i}\right) \hat{b}+\beta\left(x_{i}\right) a, \quad i=1,2, \ldots, N-2 .
\end{gathered}
$$

Since the entries of the matrix $\mathbf{D}$ depend on the grid points through the functions $\alpha(\cdot)$ and $\beta(\cdot)$ in contrast to the differentiation matrix obtained in [26] or [41], we first show two lemmas and a main result that will allow us to adapt the consistency, stability and convergence results of [26] and [41] to our setting.

Lemma 1. The function $H(\cdot)$ defined on $[0,1]$ by

$$
H(x)=\frac{S^{\prime}(x)}{S(x)},
$$

reaches a minimum and maximum given by

$$
\begin{aligned}
& \min _{x \in[0,1]} H(x)=\min \left(H\left(x^{*}\right), H(1)\right)=\left(c_{1}-c_{2}\right) C_{\min } \\
& \max _{x \in[0,1]} H(x)=\max (H(0), H(1))=\left(c_{1}-c_{2}\right) C_{\max }
\end{aligned}
$$

with $x^{*}$ a critical point of the function $H(\cdot)$ and

$$
\begin{aligned}
& c_{1}=\sinh ^{-1}\left(\zeta\left(S_{\max }-K\right)\right)(>0), \quad c_{2}=\sinh ^{-1}\left(\zeta\left(S_{\min }-K\right)\right)(<0) \\
& C_{\min }=\frac{1}{\zeta} \min \left\{\frac{\zeta}{\sqrt{1+(K \zeta)^{2}}}, \frac{\cosh \left(c_{1}\right)}{S_{\max }}\right\} \\
& C_{\max }=\frac{1}{\zeta} \max \left\{\frac{\cosh \left(c_{2}\right)}{S_{\min }}, \frac{\cosh \left(c_{1}\right)}{S_{\max }}\right\}
\end{aligned}
$$

where $\zeta$ is the stretching parameter of the transformation (8). 
Proof. For the asset price $S(x)(8)$ belonging to $\left[S_{\min }, S_{\max }\right]$ with $S_{\min }>0$ we denote

$$
S(x):=\frac{1}{\zeta} \sinh \left(c_{1} x+c_{2}(1-x)\right)+K>0,
$$

where

$$
c_{1}:=\sinh ^{-1}\left(\zeta\left(S_{\max }-K\right)\right)>0, \quad c_{2}:=\sinh ^{-1}\left(\zeta\left(S_{\min }-K\right)\right)<0
$$

since $K \in\left[S_{\min }, S_{\max }\right]$ and we easily derive, noting that $c_{1}-c_{2}>0$,

$$
S^{\prime}(x)=\frac{c_{1}-c_{2}}{\zeta} \cosh \left(c_{1} x+c_{2}(1-x)\right)>0 \quad \text { and } \quad S^{\prime \prime}(x)=\frac{\left(c_{1}-c_{2}\right)^{2}}{\zeta} \sinh \left(c_{1} x+c_{2}(1-x)\right) .
$$

Then, the extremal values for $H(x)(>0)$ solve $H^{\prime}(x)=0$, or equivalently solve $S^{\prime \prime}(x) S(x)-\left(S^{\prime}(x)\right)^{2}=0$. By substituting the first and second order derivatives (48) of $S(x)$ and using formula $\cosh ^{2} x-\sinh ^{2} x=1$, we get $\sinh \left(c_{1} x+c_{2}(1-x)\right)=1 /(K \zeta)>0$ which provides the critical points of the function $H(\cdot)$ denoted $x^{*}$. The extremal values of $H(\cdot)$ are reached in such critical points or in the boundary points $x=0$ or $x=1$. The function values of $H(\cdot)$ in those points are

$$
\begin{aligned}
& H\left(x^{*}\right)=\frac{S^{\prime}\left(x^{*}\right)}{S\left(x^{*}\right)}=\frac{\frac{c_{1}-c_{2}}{\zeta} \sqrt{1+\frac{1}{(K \zeta)^{2}}}}{\frac{1}{K \zeta^{2}}+K}=\frac{c_{1}-c_{2}}{\sqrt{1+(K \zeta)^{2}}}, \\
& H(0)=\frac{S^{\prime}(0)}{S(0)}=\frac{\left(c_{1}-c_{2}\right) \cosh \left(c_{2}\right)}{S_{\min } \zeta}, \\
& H(1)=\frac{S^{\prime}(1)}{S(1)}=\frac{\left(c_{1}-c_{2}\right) \cosh \left(c_{1}\right)}{S_{\max } \zeta} .
\end{aligned}
$$

Since $\cosh \left(c_{2}\right)>1$ and $S_{\min }<K$, it holds that $H(0)>H\left(x^{*}\right)$, and we can conclude that $\min _{x \in[0,1]} H(x)=\min \left(H\left(x^{\star}\right), H(1)\right)$ while $\max _{x \in[0,1]} H(x)=\max (H(0), H(1))$. The results then follow by substituting the found expressions of the function values $H(0), H(1)$ and $H\left(x^{\star}\right)$.

Lemma 2. The functions $\alpha(\cdot)$ and $\beta(\cdot)$ defined in (11) are bounded over $[0,1]$ as follows

$$
0<\alpha(x) \leq \frac{\sigma^{2}}{2\left(c_{1}-c_{2}\right)^{2} C_{\min }^{2}} \quad|\beta(x)| \leq \frac{\sigma^{2}}{2\left(c_{1}-c_{2}\right) C_{\min }^{2}}\left(\frac{2|r-q-\lambda \kappa|}{\sigma^{2}} C_{\min }+1\right)
$$

and, in addition,

$$
\left|\frac{\beta(x)}{\alpha(x)}\right| \leq\left(c_{1}-c_{2}\right)\left(\frac{2|r-q-\lambda \kappa|}{\sigma^{2}} C_{\max }+1\right),
$$

where $c_{1}, c_{2}, C_{\min }$ and $C_{\max }$ are defined in (45)-(47).

Proof. To bound the function $\alpha(\cdot)$ we note that we can express it in terms of the bounded function $H(\cdot)(42)$ that has minimal value (43),

$$
0<\alpha(x)=\frac{\sigma^{2}}{2} \frac{1}{H^{2}(x)} \leq \frac{\sigma^{2}}{2\left(c_{1}-c_{2}\right)^{2} C_{\min }^{2}} .
$$

Similarly we bound the function $\beta(\cdot)$ as follows

$$
|\beta(x)| \leq|r-q-\lambda \kappa| \frac{1}{\min _{[0,1]} H(x)}+\alpha(x) \max _{x \in[0,1]}\left|\frac{S^{\prime \prime}(x)}{S^{\prime}(x)}\right|,
$$

where from (48) we immediately find

$$
\left|\frac{S^{\prime \prime}(x)}{S^{\prime}(x)}\right|=\left(c_{1}-c_{2}\right)\left|\tanh \left(c_{1} x+c_{2}(1-x)\right)\right| \leq c_{1}-c_{2},
$$

which in combination with (43), (51) in (52) leads to the requested bound for $\beta(\cdot)$ in (49). Dividing the expression of $\beta(x)$ by the one of $\alpha(x)$ in (11) and recalling (42) we obtain

$$
\left|\frac{\beta(x)}{\alpha(x)}\right| \leq \frac{2|r-q-\lambda \kappa|}{\sigma^{2}} \max _{[0,1]} H(x)+\max _{x \in[0,1]}\left|\frac{S^{\prime \prime}(x)}{S^{\prime}(x)}\right| .
$$

Substituting (44) and the upperbound (53) we easily arrive at the bound (50). 
Theorem 1. The tridiagonal matrices $-\boldsymbol{D}(38)$ and $-\boldsymbol{D}+\lambda \boldsymbol{I}$ are nonsingular $M$-matrices if

$$
\frac{|r-q-\lambda \kappa|}{\sigma^{2}} \leq \frac{1}{2 C_{\max }}\left(\frac{2}{h\left(c_{1}-c_{2}\right)}\left(1+\frac{h^{2}}{2 \epsilon^{2}+h^{2}}\right)-1\right),
$$

where $\epsilon$ is the shape parameter in the RBF function (32) (with $\epsilon \gg h$ ) and $c_{1}, c_{2}$ and $C_{\max }$ are defined in (45) and (47).

Proof. We derive a sufficient condition for the matrix $-\mathbf{D}$ to be a nonsingular M-matrix by its characterization $N_{39}$ in the survey paper [36], namely the diagonal entries of $-\mathbf{D}$ are positive real numbers, the off-diagonal entries are real and nonpositive, and the matrix $-\mathbf{D}$ is strictly diagonally dominant. Since the matrix $-\mathbf{D}+\lambda \mathbf{I}$ has the same off-diagonal elements, we only have to check that its diagonal elements are positive real numbers and that the matrix is strictly diagonally dominant.

Since $r, \lambda$ and $\alpha\left(x_{i}\right)$ for all $i=1,2, \ldots, N-1$ are positive, and $b$ (37) is negative, we find for (40) that $-\mathbf{D}_{i i}=r-\alpha\left(x_{i}\right) b>0$ and $(-\mathbf{D}+\lambda \mathbf{I})_{i i}=(r+\lambda)-\alpha\left(x_{i}\right) b>0$. For the off-diagonal entries of matrix $-\mathbf{D}$, we should prove according to (41) that

$$
\beta\left(x_{i+1}\right) a-\alpha\left(x_{i+1}\right) \hat{b} \leq 0 \quad \text { and } \quad-\beta\left(x_{i}\right) a-\alpha\left(x_{i}\right) \hat{b} \leq 0, \quad i=1,2, \ldots, N-2 .
$$

Since the grid points $x_{i}$ vary with the step size $h$ we will prove these inequalities for all $x \in[0,1]$, which can be combined as

$$
-\alpha(x) \hat{b} \leq \beta(x) a \leq \alpha(x) \hat{b} \quad \Leftrightarrow \quad\left|\frac{\beta(x)}{\alpha(x)}\right| \leq \frac{\hat{b}}{a}=\frac{2}{h}\left(1+\frac{h^{2}}{2 \epsilon^{2}+h^{2}}\right),
$$

where we also used (35) and (37) in the last equality. In view of the bound (50), the latter inequality will be satisfied when

$$
\left|\frac{\beta(x)}{\alpha(x)}\right| \leq\left(c_{1}-c_{2}\right)\left(\frac{2|r-q-\lambda \kappa|}{\sigma^{2}} C_{\max }+1\right) \leq \frac{2}{h}\left(1+\frac{h^{2}}{2 \epsilon^{2}+h^{2}}\right),
$$

which is the case under condition (54), and this completes the first part of the proof.

To prove that the matrix $-\mathbf{D}$ is strictly diagonally dominant it is sufficient to show that the sum of all entries in each row is positive since the diagonal entries of $-\mathbf{D}$ are positive and the off-diagonal entries under condition (54) are nonpositive. Equivalently, we can prove that the sum of all nonzero entries of $\mathbf{D}$ in each row is negative. Starting with the first and the last row of $\mathbf{D}$, we must show that $\alpha\left(x_{1}\right)(b+\hat{b})+\beta\left(x_{1}\right) a-r<0$ and $\alpha\left(x_{N-1}\right)(b+$ $\hat{b})-\beta\left(x_{N-1}\right) a-r<0$. From (37) we know that $2 \hat{b}+b=0$. Putting $\hat{b}+b=-\hat{b}$ and using (55), we get the desired result. For the rows 2 up to $N-2$ of $\mathbf{D}$ the sum of the nonzero entries (41) equals

$$
2 \alpha\left(x_{i}\right) \hat{b}+\alpha\left(x_{i}\right) b-r=\alpha\left(x_{i}\right)(2 \hat{b}+b)-r=-r<0,
$$

where we used again that $2 \hat{b}+b=0$ and $r>0$. This completes the proof of strictly diagonal dominance for $-\mathbf{D}$. The matrix $-\mathbf{D}+\lambda \mathbf{I}$ will be a fortiori strictly diagonally dominant since a positive $\lambda$ is added to a positive sum of the row elements.

Remark 1. In the limit for h tending to zero the upper bound in (54) will tend to infinity. Hence, condition (54) in Theorem 1 is not too restrictive. For a fixed value of the shape parameter $\epsilon$ it is sufficient to choose the step size $h(\ll \epsilon)$ small enough.

\subsection{Stability analysis cf. [41]}

In [41] a stability analysis is carried out based on a linear test problem. Under Merton's and Kou's model the IMEX-midpoint scheme (29) combined with a spatial discretization is found to be conditionally stable for $\lambda \triangle \tau<1$ when a quadrature with positive weights is used for the integral term and if the eigenvalues of the differential matrix $\mathbf{D}$ are real and nonpositive, see [41, Corollary 1]. Since the matrix $-\mathbf{D}$ in the present setting is tridiagonal and an M-matrix under condition (54), it is similar to a real symmetric tridiagonal matrix and hence all its eigenvalues are real and positive (see [36, Characterization $\left.\mathrm{C}_{9}\right]$ ). In view of (27) we can conclude the following stability result.

Corollary 1. If the model parameters satisfy (54) then the discretization scheme (31) with the differential matrix $\boldsymbol{D}$ as in (38) applied to a linear test problem is conditionally stable for $\lambda \triangle \tau<1$. 


\subsection{Consistency, stability and convergence analysis cf. [26]}

In this section we adapt the consistency, stability and convergence study of [26] to our setting where we recall that the main difference is that the entries in the differentiation matrix (38) depend on the grid points while in [26] those are constants.

We introduce some additional notations as in [26]. We consider the discrete operators $\mathcal{D}_{h}, \mathcal{I}_{h}$ and $\mathcal{L}_{h}$ as approximations to the operators $\mathcal{D}, \mathcal{I}$ and $\mathcal{L}$ and defined by

$$
\begin{aligned}
\mathcal{D}_{h} u\left(\tau_{k}, x_{j}\right)=\alpha\left(x_{j}\right) \hat{b}\left(u\left(\tau_{k}, x_{j+1}\right)-2 u\left(\tau_{k}, x_{j}\right)+u\left(\tau_{k}, x_{j-1}\right)\right) & \\
& +\beta\left(x_{j}\right) a\left(u\left(\tau_{k}, x_{j+1}\right)-u\left(\tau_{k}, x_{j-1}\right)\right)-r u\left(\tau_{k}, x_{j}\right) \\
\mathcal{I}_{h} u\left(\tau_{k}, x_{j}\right)= & \lambda \frac{h}{2} \sum_{i=0}^{N} \omega_{i} F_{i}\left(x_{j}\right) u\left(\tau_{k}, x_{i}\right)+\mathcal{R}\left(\tau_{k}, x_{j}\right) \\
\mathcal{L}_{h} u\left(\tau_{k}, x_{j}\right) & =\left(\mathcal{D}_{h}-\lambda\right) \frac{u\left(\tau_{k+1}, x_{j}\right)+u\left(\tau_{k-1}, x_{j}\right)}{2}+\mathcal{I}_{h} u\left(\tau_{k}, x_{j}\right)
\end{aligned}
$$

with $\left(\tau_{k}, x_{j}\right) \in(0, T] \times(0,1)$ and where we used (24)-(27), (37), (40)-(41).

Theorem 2 (consistency). Let $u \in C^{\infty}((0, T] \times[0,1])$ satisfy the boundary and initial conditions (13)-(14). For a sufficiently small time step $\triangle \tau$ and a sufficiently small grid step size $h$, it holds that

$$
\begin{array}{r}
\frac{\partial u}{\partial \tau}\left(\tau_{k}, x_{j}\right)-\mathcal{L} u\left(\tau_{k}, x_{j}\right)-\left(\frac{u\left(\tau_{k+1}, x_{j}\right)-u\left(\tau_{k-1}, x_{j}\right)}{2 \triangle \tau}-\mathcal{L}_{h} u\left(\tau_{k}, x_{j}\right)\right)=O\left((\triangle \tau)^{2}+h^{2}+\frac{h^{2}}{\epsilon^{2}}\right) \\
1 \leq k \leq M-1
\end{array}
$$

where $\left(\tau_{k}, x_{j}\right) \in(0, T] \times(0,1)$ and $\epsilon$ is the shape parameter in the RBF function (32) (with $\left.\epsilon \gg h\right)$.

Proof. The proof follows the same steps as the proof of [26, Theorem 6.1]. We will only discuss in detail the steps that differ. As can be seen from section 4.2 the error for approximating the integral operator originates from the error of applying the trapezoidal rule leading to

$$
\mathcal{I} u\left(\tau_{k}, x_{j}\right)-\mathcal{I}_{h} u\left(\tau_{k}, x_{j}\right)=O\left(h^{2}\right) .
$$

The error for the approximation of the first-order derivative in time is by Taylor's expansion $O\left((\triangle \tau)^{2}\right)$ for $1 \leq$ $k \leq M-1$. Also the interpolation error formula in time for the first and second order partial derivative with respect to $x$ is $O\left((\triangle \tau)^{2}\right)$. The errors originating from the RBF-FD approximation to the first and second order partial derivatives with respect to $x$ are derived in [5], providing

$$
\begin{aligned}
& \frac{\partial u}{\partial x}\left(\tau_{k}, x_{j}\right)-a\left(u\left(\tau_{k}, x_{j+1}\right)-u\left(\tau_{k}, x_{j-1}\right)\right)=O\left(h^{2}+\frac{h^{2}}{\epsilon^{2}}\right), \\
& \frac{\partial^{2} u}{\partial x^{2}}\left(\tau_{k}, x_{j}\right)-\hat{b}\left(u\left(\tau_{k}, x_{j+1}\right)-2 u\left(\tau_{k}, x_{j}\right)+u\left(\tau_{k}, x_{j-1}\right)\right)=O\left(h^{2}+\frac{h^{2}}{\epsilon^{2}}\right) .
\end{aligned}
$$

Combining these errors as in [26] and noting that the functions $\alpha(\cdot)$ and $\beta(\cdot)$ are bounded over $[0,1]$ by constants independent of $h$ and of $\triangle \tau$, see (49), we obtain that the differential operator $\mathcal{D}$ is approximated by the discrete operator $\mathcal{D}_{h}$ with the error at each mesh point $\left(\tau_{k}, x_{j}\right)$

$$
\mathcal{D} u\left(\tau_{k}, x_{j}\right)-\mathcal{D}_{h} \frac{u\left(\tau_{k+1}, x_{j}\right)+u\left(\tau_{k-1}, x_{j}\right)}{2}=O\left((\triangle \tau)^{2}+h^{2}+\frac{h^{2}}{\epsilon^{2}}\right) .
$$

In addition we need the following interpolation error when $1 \leq k \leq M-1$

$$
\left|u\left(\tau_{k}, x_{j}\right)-\frac{1}{2}\left(u\left(\tau_{k+1}, x_{j}\right)+u\left(\tau_{k-1}, x_{j}\right)\right)\right| \leq \frac{(\triangle \tau)^{2}}{2} \sup _{\tau \in\left[\tau_{k-1}, \tau_{k+1}\right]}\left|\frac{\partial^{2} u}{\partial \tau^{2}}\left(\tau, x_{j}\right)\right|,
$$

to finally arrive at the stated error.

Theorem 3 (stability). The RBF-FD scheme (28)-(29) is stable in the sense of the von Neumann analysis if $\lambda \triangle \tau \leq 1 / 2$. 
Proof. This proof follows the same lines as the proof of [26, Theorem 6.3] and the stability is proved by using [44, Theorem 4.2.2].

For a fixed time step $k, 1 \leq k \leq M-1$, the $j$ th equation in the scheme (29) can be expressed in terms of the introduced discrete operators as follows

$$
\frac{U_{j}^{k+1}-U_{j}^{k-1}}{2 \triangle \tau}=\left(\mathcal{D}_{h}-\lambda\right) \frac{U_{j}^{k+1}+U_{j}^{k-1}}{2}+\mathcal{I}_{h} U_{j}^{k} .
$$

Multiplying both sides by $2 \triangle \tau$, neglecting the last term $\mathcal{R}\left(\tau_{k}, x_{j}\right)$ which does not contain $U_{j}^{k}$, substituting $g^{k} \mathrm{e}^{\mathrm{i} j \theta}$ for $U_{j}^{k}$ and dividing both sides of the resulting relation by $g^{k-1} \mathrm{e}^{\mathrm{i} j \theta}$, we obtain the amplification polynomial in the von Neumann analysis defined by

$$
\Phi(\theta, \triangle \tau, h)=\beta_{2} g^{2}-2 \beta_{1} g-\beta_{0},
$$

where the coefficients depend on the grid points $x_{j}$ and are given by

$$
\begin{aligned}
& \beta_{2}\left(x_{j}\right)=1+\triangle \tau\left(2 \alpha\left(x_{j}\right) \hat{b}(1-\cos \theta)+(r+\lambda)\right)-\mathrm{i} 2 \triangle \tau \beta\left(x_{j}\right) a \sin \theta \\
& \beta_{1}\left(x_{j}\right)=\lambda \triangle \tau\left(\frac{h}{2} \sum_{n=1}^{N-1} \omega_{n} F_{n}\left(x_{j}\right) \mathrm{e}^{\mathrm{i}(n-j) \theta}\right) \\
& \beta_{0}\left(x_{j}\right)=1-\triangle \tau\left(2 \alpha\left(x_{j}\right) \hat{b}(1-\cos \theta)+(r+\lambda)\right)+\mathrm{i} 2 \triangle \tau \beta\left(x_{j}\right) a \sin \theta .
\end{aligned}
$$

Since the function $\alpha(\cdot)$ is strictly positive over [0,1], see (49), and $\hat{b}>0$ (37) it is clear that $\left|\beta_{2}\left(x_{j}\right)\right|>1$ and $\left|\beta_{0}\left(x_{j}\right) / \beta_{2}\left(x_{j}\right)\right|<1$ for $j=1, \ldots, N-1$. Further, since we are dealing with a density function it holds that $\left|\beta_{1}\left(x_{j}\right)\right| \leq \lambda \triangle \tau$. Thus, we have (when omitting the dependence on $x_{j}$ to simplify the notations)

$$
|g|=\left|\frac{\beta_{1} \pm \sqrt{\beta_{1}^{2}-\beta_{2} \beta_{0}}}{\beta_{2}}\right| \leq 2\left|\frac{\beta_{1}}{\beta_{2}}\right|+\left|\frac{\beta_{0}}{\beta_{2}}\right|^{1 / 2} \leq 1+2 \lambda \triangle \tau,
$$

which proves (a) in [44, Theorem 4.2.2]. To prove [44, Theorem 4.2.2 (b)] we note that the sum of the roots $g_{1}$ and $g_{2}$ of $\Phi(\theta, \triangle \tau, h)$ satisfies

$$
\left|g_{1}+g_{2}\right|=2\left|\beta_{1} / \beta_{2}\right| \leq 2 \lambda \triangle \tau
$$

and hence if $\left|g_{1}\right|$ is larger than 1 , then

$$
\left|g_{2}-g_{1}\right| \geq 2\left|g_{1}\right|-\left|g_{1}+g_{2}\right| \geq 2-2 \lambda \triangle \tau \geq 1
$$

holds when $\lambda \triangle \tau \leq 1 / 2$.

To prove the global convergence of the scheme we introduce the error vectors at time level $k, 1 \leq k \leq M$,

$$
\xi^{k}:=\left[\begin{array}{llll}
\xi_{1}^{k} & \xi_{2}^{k} & \cdots & \xi_{N-1}^{k}
\end{array}\right]^{\top} \quad \text { with } \quad \xi_{j}^{k}=u\left(\tau_{k}, x_{j}\right)-U_{j}^{k}, \quad j=1, \ldots, N-1 .
$$

The boundary errors $\xi_{0}^{k}$ and $\xi_{N}^{k}$ are zero by the imposed boundary conditions in section 4.4.

We further define the following vector norms. Let $\mathbb{R}^{N}$ be the space of column vectors of dimension $N$ with real entries. For a given column vector $\mathbf{x}=\left[\begin{array}{llll}x_{1} & x_{2} & \cdots & x_{N}\end{array}\right]^{\top} \in \mathbb{R}^{N}$ we introduce two discrete vector norms $\|\cdot\|_{\ell^{2}}$ and $\|\cdot\|_{\ell^{\infty}}$ defined by

$$
\|\mathbf{x}\|_{\ell^{2}}=\left(h \sum_{j=1}^{N} x_{j}^{2}\right)^{\frac{1}{2}}, \quad\|\mathbf{x}\|_{\ell^{\infty}}=\max _{1 \leq j \leq N}\left|x_{j}\right|,
$$

and two induced matrix norms $\|\mathbf{A}\|_{2}$ and $\|\mathbf{A}\|_{\infty}$ defined by

$$
\|\mathbf{A}\|_{2}=\max _{\mathbf{x} \neq \mathbf{0}} \frac{\|\mathbf{A} \mathbf{x}\|_{\ell^{2}}}{\|\mathbf{x}\|_{\ell^{2}}}, \quad\|\mathbf{A}\|_{\infty}=\max _{\mathbf{x} \neq \mathbf{0}} \frac{\|\mathbf{A} \mathbf{x}\|_{\ell^{\infty}}}{\|\mathbf{x}\|_{\ell^{\infty}}}=\max _{i}\left(\sum_{j=1}^{N}\left|a_{i j}\right|\right) .
$$


Theorem 4 (convergence). Let $u \in C^{\infty}((0, T] \times[0,1])$ be the solution to the initial-valued PIDE in (9)-(14) and let $\mathbf{U}^{k}$ be the solution to the RBF-FD approximation in (28)-(29). Assume that $\boldsymbol{B}+\boldsymbol{B}^{\top}$ is positive semi-definite with $\boldsymbol{B}=-2(\boldsymbol{D}-\lambda \boldsymbol{I})$. If $\triangle \tau$ and $h$ are sufficiently small and condition (54) is satisfied, then there exists a positive constant $C$ independent of $\triangle \tau$ and $h$ such that for $1 \leq k \leq M-1$

$$
\left\|\boldsymbol{\xi}^{k}\right\|_{\ell^{2}} \leq C\left((\triangle \tau)^{2}+h^{2}+\frac{h^{2}}{\epsilon^{2}}\right)
$$

where $\epsilon$ is the shape parameter in the RBF function (32) (with $\epsilon \gg h$ ).

Proof. This proof follows the same lines as the one of [26, Theorem 6.5]. Therefore we will only indicate how that reasoning can be applied to our setting and point out the differences.

We start by rewriting (29) as

$$
(\mathbf{I}-\triangle \tau(\mathcal{D}-\lambda \mathbf{I})) \mathbf{U}^{k+1}=(\mathbf{I}+\triangle \tau(\mathcal{D}-\lambda \mathbf{I})) \mathbf{U}^{k-1}+2 \lambda \triangle \tau \mathcal{J} \mathbf{U}^{k}+2 \lambda \triangle \tau \mathcal{R}^{k}, \quad \text { for } 1 \leq k \leq M-1,
$$

with the matrices $\mathcal{J}$ and $\mathcal{R}^{k}$ defined in that subsection. Combination with Theorem 2 and the boundary conditions provides in matrix form the following recursion for the error vector

$$
\left(\mathbf{I}+\frac{\triangle \tau}{2} \mathbf{B}\right) \boldsymbol{\xi}^{k+1}=\left(\mathbf{I}-\frac{\triangle \tau}{2} \mathbf{B}\right) \boldsymbol{\xi}^{k-1}+2 \lambda \triangle \tau \mathbf{J} \boldsymbol{\xi}^{k}+\mathbf{d}, \quad 1 \leq k \leq M-1
$$

where $\mathbf{I}$ is the identity matrix of order $N-1, \mathbf{J}=\left(\frac{\triangle z}{2} \omega_{j} F_{j}\left(x_{i}\right)\right)_{1 \leq i, j \leq N-1}$ is a submatrix of $\mathcal{J}$,

$$
\mathbf{B}=-2(\mathbf{D}-\lambda \mathbf{I}) \quad \text { and } \quad \mathbf{d}=\left[\begin{array}{llll}
d_{1} & d_{2} & \cdots & d_{N-1}
\end{array}\right]^{\top} \text { with } d_{j}=\Delta \tau O\left((\triangle \tau)^{2}+h^{2}+\frac{h^{2}}{\epsilon^{2}}\right) .
$$

For a sufficiently small space step size $h$, it was proven in Theorem 1 that $-(\mathbf{D}-\lambda \mathbf{I})$, and hence for a positive $\triangle \tau$ also $-2 \triangle \tau(\mathbf{D}-\lambda \mathbf{I})$, is a nonsingular M-matrix. Characterization $\mathbf{C}_{10}$ in [36] guarantees that $\left(\mathbf{I}+\frac{\triangle \tau}{2} \mathbf{B}\right)$ is nonsingular and its inverse exists. Further, the symmetric matrix $\mathbf{B}+\mathbf{B}^{\top}$ is positive semi-definite by assumption and the estimates $[26,(6.22)]$ and $[26,(6.23)]$ also hold in that case, namely

$$
\left\|\left(\mathbf{I}+\frac{\triangle \tau}{2} \mathbf{B}\right)^{-1}\left(\mathbf{I}-\frac{\triangle \tau}{2} \mathbf{B}\right)\right\|_{2} \leq 1, \quad\left\|\left(\mathbf{I}+\frac{\triangle \tau}{2} \mathbf{B}\right)^{-1}\right\|_{2} \leq 1 .
$$

As a next step we show that for some constant $c(>0)$ independent of $\triangle \tau$ and $h$

$$
\left\|\left(\mathbf{I}+\frac{\triangle \tau}{2} \mathbf{B}\right)^{-1} \mathbf{J}\right\|_{2} \leq c
$$

Hereto we cannot follow the approach of [26] since our matrix $\mathbf{J}$ is not a Toeplitz matrix. We proceed as follows, using (62),

$$
\begin{aligned}
\left\|\left(\mathbf{I}+\frac{\triangle \tau}{2} \mathbf{B}\right)^{-1} \mathbf{J}\right\|_{2} \leq\|\mathbf{J}\|_{2} & \leq \sqrt{\left\|\mathbf{J}^{T} \mathbf{J}\right\|_{\infty}} \\
& =\sqrt{\left\|\mathbf{J}^{T} \mathbf{H} \mathbf{H}^{-1} \mathbf{J}\right\|_{\infty}} \\
& \leq \sqrt{\left\|\mathbf{J}^{T} \mathbf{H}\right\|_{\infty}\left\|\mathbf{H}^{-1}\right\|_{\infty}\|\mathbf{J}\|_{\infty}},
\end{aligned}
$$

where

$$
\mathbf{H}:=\operatorname{diag}\left(H\left(x_{1}\right), H\left(x_{2}\right), \ldots, H\left(x_{N-1}\right)\right) .
$$

Invoking the definition (42) of function $H(\cdot)$ and of the infinity norm (59) and using (43) we find

$$
\left\|\mathbf{H}^{-1}\right\|_{\infty}=\max _{1 \leq i \leq N-1}\left|\left(H\left(x_{i}\right)\right)^{-1}\right|=\left(\min _{1 \leq i \leq N-1}\left|H\left(x_{i}\right)\right|\right)^{-1} \leq\left(\min _{x \in[0,1]}|H(x)|\right)^{-1}=\left(\left(c_{1}-c_{2}\right) C_{\min }\right)^{-1}
$$


with $c_{1}, c_{2}$ and $C_{\min }$ given by (45)-(46).

Next, we derive a bound for

$$
\left\|\mathbf{J}^{T} \mathbf{H}\right\|_{\infty}=\max _{1 \leq i \leq N-1} \frac{\triangle z}{2} \omega_{i} \sum_{j=1}^{N-1} F_{i}\left(x_{j}\right) H\left(x_{j}\right),
$$

where, recalling the definition of $F_{i}\left(x_{j}\right)$,

$$
\sum_{j=1}^{N-1} F_{i}\left(x_{j}\right) H\left(x_{j}\right)=\sum_{j=1}^{N-1} f\left(\frac{S\left(z_{i}\right)}{S\left(x_{j}\right)}\right) \frac{S^{\prime}\left(z_{i}\right)}{S\left(x_{j}\right)} \frac{S^{\prime}\left(x_{j}\right)}{S\left(x_{j}\right)}=S^{\prime}\left(z_{i}\right) \sum_{j=1}^{N} f\left(\frac{S\left(z_{i}\right)}{S\left(x_{j}\right)}\right) \frac{S^{\prime}\left(x_{j}\right)}{\left(S\left(x_{j}\right)\right)^{2}} .
$$

Then, taking into account that $f(\cdot)$ is a density function, that $S^{\prime}(x)(48)$ is positive and adding the terms with the integration boundary points and applying the substitution $y:=S\left(z_{i}\right) / S(x)$ we find

$$
\begin{aligned}
\frac{\triangle x}{2} \sum_{j=1}^{N-1} \omega_{j} f\left(\frac{S\left(z_{i}\right)}{S\left(x_{j}\right)}\right) \frac{S^{\prime}\left(x_{j}\right)}{\left(S\left(x_{j}\right)\right)^{2}} & \leq-\int_{0}^{1} f\left(\frac{S\left(z_{i}\right)}{S(x)}\right)\left(-\frac{S^{\prime}(x)}{(S(x))^{2}}\right) \mathrm{d} x=\frac{1}{S\left(z_{i}\right)} \int_{\frac{S\left(z_{i}\right)}{S_{\max }}}^{\frac{S\left(z_{i}\right)}{S_{\min }}} f(y) \mathrm{d} y \\
& \leq \frac{1}{S\left(z_{i}\right)} \int_{0}^{\infty} f(y) \mathrm{d} y=\frac{1}{S\left(z_{i}\right)} .
\end{aligned}
$$

Since $\triangle z=\triangle x=h$ and $\omega_{i}=\omega_{j}=2$ for $1 \leq i, j \leq N-1$, combination of the estimates above leads to

$$
\frac{\triangle z}{2} \omega_{i} \sum_{j=1}^{N-1} F_{i}\left(x_{j}\right) H\left(x_{j}\right)=\frac{h}{2} \sum_{j=1}^{N-1} \omega_{j} F_{i}\left(x_{j}\right) H\left(x_{j}\right) \leq \frac{S^{\prime}\left(z_{i}\right)}{S\left(z_{i}\right)}=H\left(z_{i}\right),
$$

and by using (44)

$$
\left\|\mathbf{J}^{T} \mathbf{H}\right\|_{\infty} \leq \max _{1 \leq i \leq N-1} H\left(z_{i}\right)=\|\mathbf{H}\|_{\infty} \leq\left(c_{1}-c_{2}\right) C_{\max } .
$$

Finally, substituting the estimations (65) and (66) in (64) and since $\|\mathbf{J}\|_{\infty} \leq 1$, we get the required estimate (63) with $c=\sqrt{C_{\max } / C_{\min }}$ where $C_{\min }$ and $C_{\max }$ are independent of $\Delta \tau$ and $h$ as can be seen from (46)-(47). Hence, we obtain from (61) a recursion for the error vector in the discrete vector norm

$$
\left\|\boldsymbol{\xi}^{k+1}\right\|_{\ell^{2}} \leq\left\|\boldsymbol{\xi}^{k-1}\right\|_{\ell^{2}}+2 c \lambda \triangle \tau\left\|\boldsymbol{\xi}^{k}\right\|_{\ell^{2}}+\|\boldsymbol{d}\|_{\ell^{2}} .
$$

Applying [26, Lemma 6.4] with initial condition $\left\|\xi^{0}\right\|_{\ell^{2}}=0$, we get

$$
\begin{aligned}
\left\|\boldsymbol{\xi}^{k+1}\right\|_{\ell^{2}} & \leq(1+2 c \lambda \triangle \tau)^{k}\left\|\boldsymbol{\xi}^{1}\right\|_{\ell^{2}}+\|\boldsymbol{d}\|_{\ell^{2}} \sum_{j=0}^{k-1}(1+2 c \lambda \triangle \tau)^{j} \\
& \leq \mathrm{e}^{2 c \lambda T}\left\|\boldsymbol{\xi}^{1}\right\|_{\ell^{2}}+\frac{\mathrm{e}^{2 c \lambda T}-1}{2 c \lambda \triangle \tau}\|\boldsymbol{d}\|_{\ell^{2}}
\end{aligned}
$$

where $\left\|\boldsymbol{\xi}^{1}\right\|_{\ell^{2}}=\triangle \tau O\left(\triangle \tau+h^{2}+h^{2} / \epsilon^{2}\right)$ and $\|\boldsymbol{d}\|_{\ell^{2}} \leq \triangle \tau O\left((\triangle \tau)^{2}+h^{2}+h^{2} / \epsilon^{2}\right)$, hence providing the stated result (60).

Remark 2. The positive semi-definiteness of the matrix $\boldsymbol{B}+\boldsymbol{B}^{\top}$ will be tested in the numerical experiments by checking whether $\mu_{\min }\left(\boldsymbol{B}+\boldsymbol{B}^{\top}\right) \geq 0$, implying that all eigenvalues of $\boldsymbol{B}+\boldsymbol{B}^{\top}$ are positive. For all the parameter cases that we study in section 7, we even found that all eigenvalues of $\boldsymbol{B}+\boldsymbol{B}^{\top}$ are strictly positive. It is possible to derive a relation in terms of first and second order derivatives of the functions $\alpha$ and $\beta$ in (11) that implies the positive (semi-)definiteness. However, this involved condition has also to be tested numerically for the different parameter cases while the computation of the minimal eigenvalue of $\boldsymbol{B}+\boldsymbol{B}^{\top}$ is immediate in MATLAB.

\section{Greeks}

In the numerical experiments we will also compute the option Greeks Delta and Gamma which are important for hedging the option. Delta measures the sensitivity of the option price to a fluctuation in the underlying asset price 
while Gamma is the speed at which the Delta changes with respect to this movement. They are defined as $\Delta=\frac{\partial V}{\partial S}$ and $\Gamma=\frac{\partial^{2} V}{\partial S^{2}}$ respectively, where by the chain rule we get

$$
\begin{gathered}
\Delta=\frac{\partial V}{\partial S}(\tau, S(x))=\frac{\partial u}{\partial x}(\tau, x) \frac{\mathrm{d} x}{\mathrm{~d} S}=\frac{1}{S^{\prime}(x)} \frac{\partial u}{\partial x}(\tau, x), \\
\Gamma=\frac{\partial^{2} V}{\partial S^{2}}(\tau, S(x))=\frac{\partial \Delta}{\partial S}=\frac{1}{\left(S^{\prime}(x)\right)^{2}} \frac{\partial^{2} u}{\partial x^{2}}(\tau, x)-\frac{1}{\left(S^{\prime}(x)\right)^{3}} S^{\prime \prime}(x) \frac{\partial u}{\partial x}(\tau, x) .
\end{gathered}
$$

At the time level $\tau_{M}$ we need to compute the $\Delta$ and $\Gamma$ functions at the grid points $S_{0}, S_{1}, \cdots, S_{N}$ corresponding to grid points in the set $X=\left\{x_{0}, x_{1}, \cdots, x_{N}\right\}$. By means of the differential matrices $\mathbf{D}_{\mathbf{x}}$ and $\mathbf{D}_{\mathbf{x x}}$ of order $N+1$, (39), we derive the following approximations for $\Delta$ and $\Gamma$,

$$
\begin{aligned}
\Delta^{M} & =\frac{1}{\mathbf{S}^{\prime}(\mathbf{x})} \mathbf{D}_{\mathbf{x}} \mathbf{U}^{M} \\
\Gamma^{M} & =\left(\frac{1}{\mathbf{S}^{\prime}(\mathbf{x})}\right)^{2} \mathbf{D}_{\mathbf{x x}} \mathbf{U}^{M}-\left(\frac{1}{\mathbf{S}^{\prime}(\mathbf{x})}\right)^{3} \mathbf{S}^{\prime \prime}(\mathbf{x}) \mathbf{D}_{\mathbf{x}} \mathbf{U}^{M}
\end{aligned}
$$

with $\mathbf{U}^{M}$ the solution to the fully discretized problem (28)-(29) in the European option case and (30)-(31) in the American option case, and with notations

$$
\begin{aligned}
\left(\frac{1}{\mathbf{S}^{\prime}(\mathbf{x})}\right)^{\ell} & :=\operatorname{diag}\left(\frac{1}{\left(S^{\prime}\left(x_{0}\right)\right)^{\ell}}, \frac{1}{\left(S^{\prime}\left(x_{1}\right)\right)^{\ell}}, \ldots, \frac{1}{\left(S^{\prime}\left(x_{N}\right)\right)^{\ell}}\right), \quad \ell=1,2,3, \\
\mathbf{S}^{\prime \prime}(\mathbf{x}) & :=\operatorname{diag}\left(S^{\prime \prime}\left(x_{0}\right), S^{\prime \prime}\left(x_{1}\right), \ldots, S^{\prime \prime}\left(x_{N}\right)\right),
\end{aligned}
$$

where $S^{\prime}(x)$ and $S^{\prime \prime}(x)$ are given in (48).

\section{Numerical Results}

In this section, we report on the performance of our proposed method to price European and American options under the Merton model and the Kou model by carrying out several numerical simulations on a PC Laptop with an Intel(R) $3.6 \mathrm{GHz}$ Core i3 processor. The software programs are written in MATLAB.

The $N+1$ collocation points $x_{j}, j=0, \ldots, N$ in the spatial discretization are chosen to be uniform in the interval $[0,1]$. The corresponding values (8) for $S$ are more concentrated around the strike price $K$ as can be seen in Figure 1.
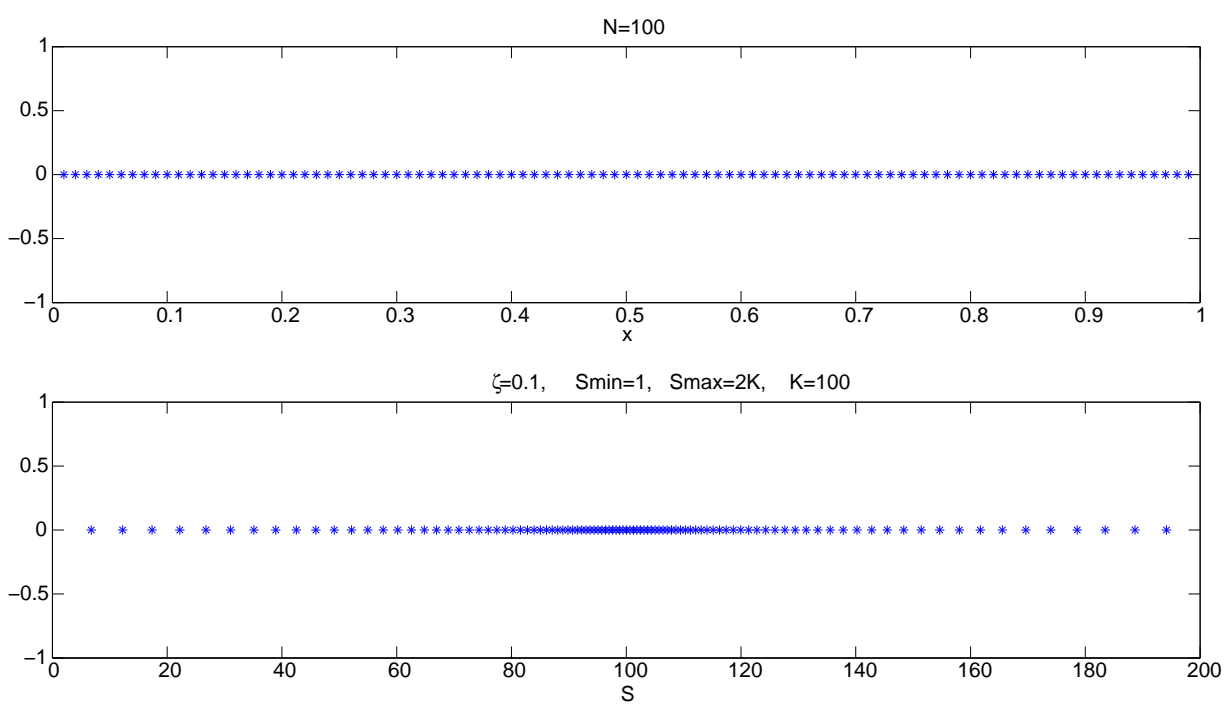

Figure 1: Uniform grid for $x$ and corresponding non-uniform grid for $S$ 
Although the proposed scheme works for all radial basis functions from Table 1 we choose the multiquadric radial basis function (32) for all the numerical experiments. The accuracy of RBF-methods where the differentiation matrix contains the differentiations of the shape function highly depends upon the shape parameter $\epsilon$ of the basis functions, which is responsible for the flatness of the functions. For smooth problems, the best accuracy is typically achieved when $\epsilon$ is small, but then the condition number of the linear system becomes very large. In [19] they show the error convergence according to the variation of the shape parameter. In the RBF-FD method the shape parameter enters the weights in the approximation of the derivatives as can be seen in (35) and (37). In [23] a different shape parameter is chosen for each case that is studied in the numerical simulations. It turns out that our proposed RBF-FD method is rather robust with respect to the shape parameter in the sense that we can use one fixed value for $\epsilon$ for all cases in our numerical study. Hereto, we try to find the best compromise for the size of $\epsilon$ by means of the root mean square (RMS) error defined by

$$
\text { RMS error }=\sqrt{\frac{1}{3} \sum_{j=1}^{3}\left(U_{j}^{M}-V\left(0, S_{j}\right)\right)^{2}},
$$

where $U_{j}^{M}$ is an RBF-FD based approximation from section 4.3 or section 4.4 for the exact option price $V\left(0, S_{j}\right)$ with $S_{j} \in\{90,100,110\}$. For European options the exact prices $V\left(0, S_{j}\right), j=1,2,3$, are available and presented in Table 5. For American options the exact prices are not available and we use the reference values from Table 5 to evaluate this RMS error. For both European and American options, we compute an RBF-FD solution $U_{j}^{M}$ with $N=256$ and $M=128$. Figure 2 displays the dependence of this root mean square error on the size of

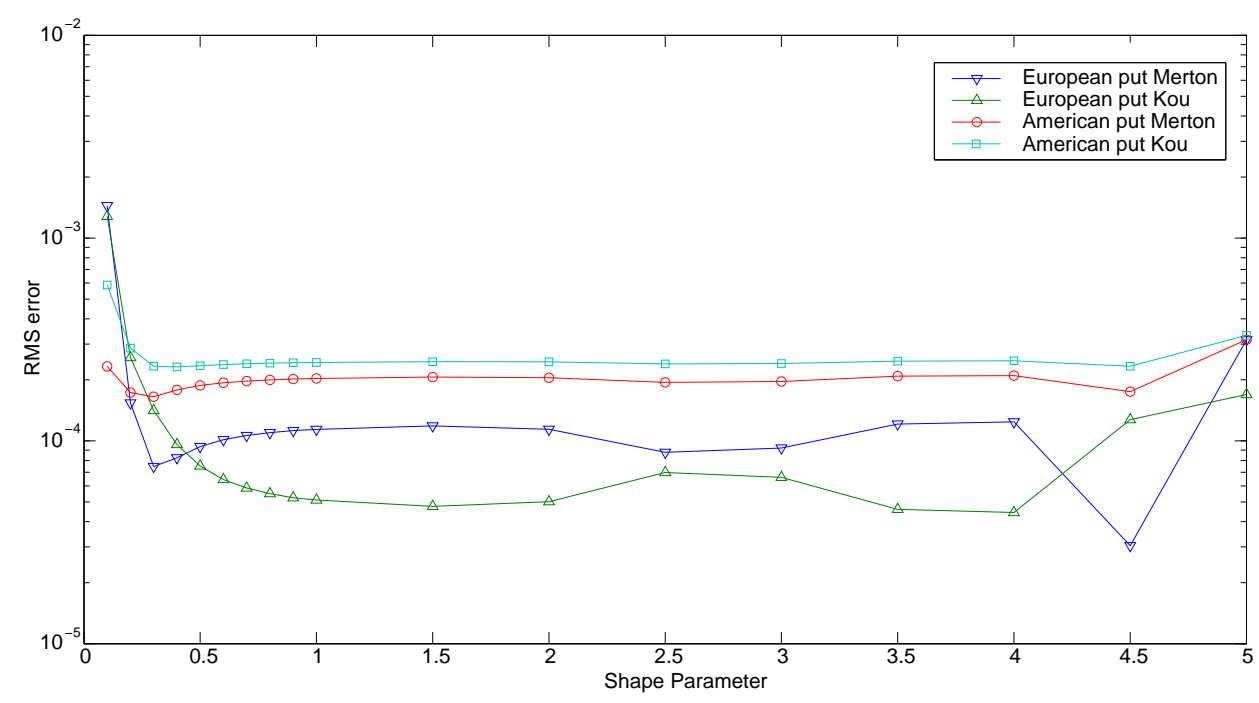

Figure 2: Root mean square (RMS) error as function of the shape parameter $\epsilon$ for European and American options and parameter set 1

the shape parameter for European and American options for some common test examples. We derive from it that $\epsilon=1$ is a good choice for our numerical experiments. In what follows we fix $\epsilon$ on this value, thus we work with the multiquadric radial basis function $\phi(|x-y|)=\sqrt{1+|x-y|^{2}}$.

\subsection{Convergence rate for European and American option prices under Merton and Kou model}

We will study numerically the convergence rate of the proposed method using the root mean square error as defined in (68). The jump distribution parameters are not changed between experiments and are given in Table 2. The shape parameter $\epsilon$ in the RBF is fixed on 1 as motivated above.

In the numerical experiments we use reference prices for the two parameter sets from Table 3 as the exact prices in (68). The first parameter set is as reported in [1] while the second one is taken from [40, 37]. In Table 4 we list for which maximal value of $h$ and minimal value $N=1 / h$ condition (54) is satisfied for the different models. The reference prices are listed in Table 5. As already mentioned, the European call and put option prices 


\begin{tabular}{lccccc}
\hline & $\mu$ & $\gamma$ & $\alpha_{1}$ & $\alpha_{2}$ & $p$ \\
\hline $\begin{array}{l}\text { Merton } \\
\text { Kou }\end{array}$ & -0.9 & 0.45 & & & \\
\hline
\end{tabular}

Table 2: Jump distribution parameters

can be computed exactly using the analytical formula in [30] for the Merton model and in [25] for the Kou model. For the American put option prices we will use results from the literature as benchmark values. For the Merton model the reference prices with the first set of parameters are taken from [13] which were also used as reference values in e.g. [27, 40, 9, 23, 37] while those with the second set of parameters are as in [40, 37]. For the Kou model and the first parameter set we consider the prices reported in [45] and used in e.g. [39, 27, 40, 23, 37]. Reference American put option prices under the Kou model with the second parameter set are again taken from [40, 37].

\begin{tabular}{lcccccc}
\hline & $\sigma$ & $r$ & $q$ & $T$ & $K$ & $\lambda$ \\
\hline parameter set 1 & 0.15 & 0.05 & 0 & 0.25 & 100 & 0.1 \\
parameter set 2 & 0.1 & 0.1 & 0 & 1 & 100 & 0.5 \\
\hline
\end{tabular}

Table 3: Model parameters and data

\begin{tabular}{lccccc}
\hline & $S_{\min }$ & $S_{\max }$ & $\zeta$ & $N$ & $h$ \\
\hline Merton, parameter set 1 & $0.03 \mathrm{~K}$ & $2 \mathrm{~K}$ & $7 / \mathrm{K}$ & 800 & 0.001251 \\
Merton, parameter set 2 & $0.3 \mathrm{~K}$ & $4 \mathrm{~K}$ & $6 / \mathrm{K}$ & 542 & 0.001848 \\
Kou, parameter set 1 & $0.3 \mathrm{~K}$ & $2 \mathrm{~K}$ & $6 / \mathrm{K}$ & 27 & 0.038461 \\
Kou, parameter set 2 & $0.3 \mathrm{~K}$ & $4 \mathrm{~K}$ & $7 / \mathrm{K}$ & 142 & 0.007092 \\
\hline
\end{tabular}

Table 4: Maximal value for $h$ and minimal value $N$ so that condition (54) is satisfied
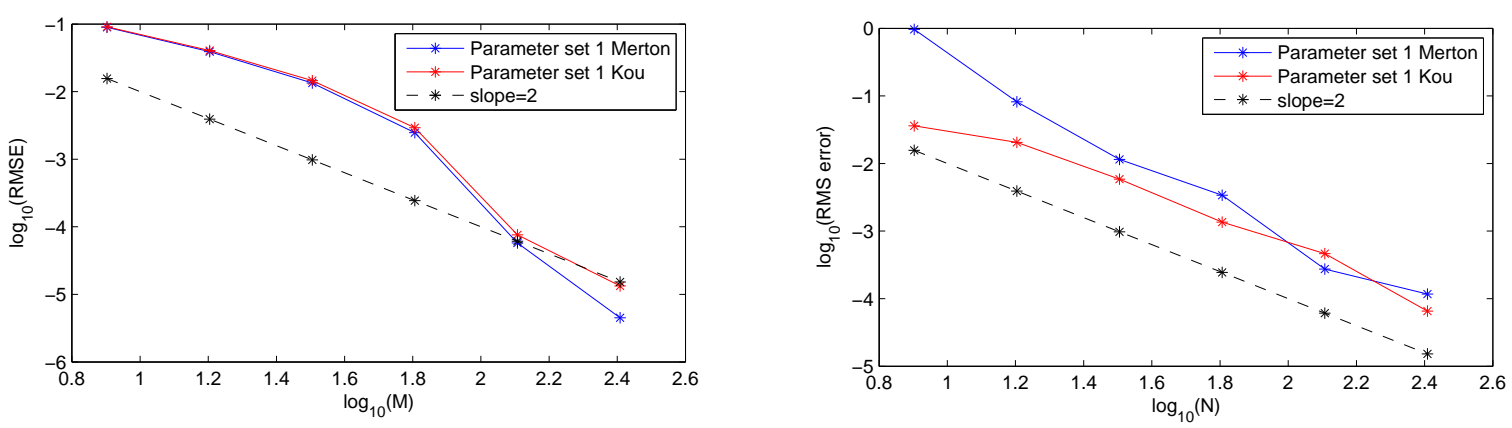

Figure 3: European put option for parameter set 1. Left: Error convergence in time using 512 nodal points. Right: Error convergence in space using 512 time discretization steps.

From Figures 3-4 we may conclude that for both European and American put option prices and in both jumpdiffusion models for different parameter sets the proposed method is second order in time and space. This is in accordance with the result in Theorem 4. 


\begin{tabular}{lccc}
\hline Model, type & $S=90$ & $S=100$ & $S=110$ \\
\hline parameter set 1 & & & \\
Merton, European call & 0.527638 & 4.391246 & 12.643406 \\
Merton, European put & 9.285418 & 3.149026 & 1.401186 \\
Merton, American put & 10.003822 & 3.241251 & 1.419803 \\
Kou, European call & 0.672677 & 3.973479 & 11.794583 \\
Kou, European put & 9.430457 & 2.731259 & 0.552363 \\
Kou, American put & 10.005071 & 2.807879 & 0.561876 \\
parameter set 2 & & & \\
Merton, American put & 19.948906 & 18.246332 & 16.666925 \\
Kou, American put & 10.698208 & 6.417275 & 4.624099 \\
\hline
\end{tabular}

Table 5: Reference prices used in numerical experiments
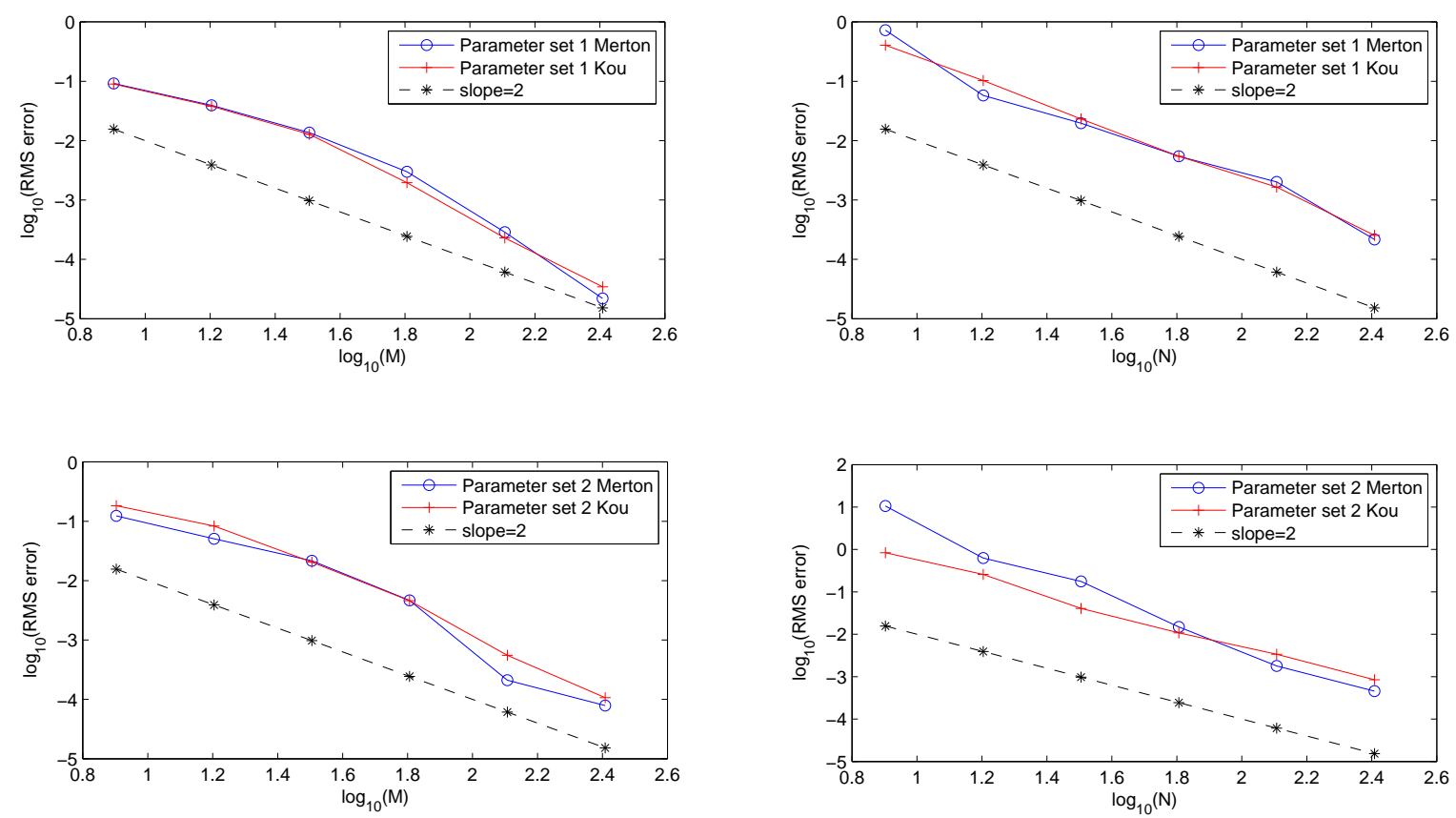

Figure 4: American put option for parameter set 1 respectively parameter set 2. Left: Error convergence in time using 512 nodal points. Right: Error convergence in space using 512 time discretization steps.

\subsection{Comparison to other methods}

The goal of this section is to compare the accuracy and efficiency of the proposed method to other schemes in the literature. Standard we will report in the tables the absolute error between the computed option price and the reference price unless otherwise stated. In the numerical experiments we will use some other parameter sets than those in Table 3, see Table 6.

Comparison to other RBF-methods for European option prices under the Merton model In [38] a numerical test is carried out to compare the radial basis function scheme based on differential quadrature (RBF-DQ) against a traditional finite difference (FD) approximation of the pricing PIDE. We do the same test with our proposed RBF-FD method. Recall that the RBF-DQ method leads to a full differentiation matrix since the RBFs are evaluated at all spatial discretization nodes while the RBF-FD method results in a sparse differentiation matrix. All three schemes put $N=512$ for the spatial nodes and evaluate an European call option price under the Merton 


\begin{tabular}{lccccccccc}
\hline & $\sigma$ & $r$ & $q$ & $T$ & $K$ & $\lambda$ & $S_{\min }$ & $S_{\max }$ & $\zeta$ \\
\hline parameter set 3 & 0.2 & 0.1 & 0 & 3 & 1 & 0.1 & $0.01 \mathrm{~K}$ & $4 \mathrm{~K}$ & $6 / \mathrm{K}$ \\
parameter set 4 & 0.35 & 0.05 & 0 & 1 & 1 & 0.1 & $0.01 \mathrm{~K}$ & $4 \mathrm{~K}$ & $6 / \mathrm{K}$ \\
parameter set 5 & $0.1 / 1$ & 0.03 & 0 & 0.1 & 1 & 0.1 & $0.1 \mathrm{~K}$ & $4 \mathrm{~K}$ & $6 / \mathrm{K}$ \\
parameter set 6 & 0.2 & 0.05 & 0 & 3 & 100 & 0.2 & $0.1 \mathrm{~K}$ & $4 \mathrm{~K}$ & $6 / \mathrm{K}$ \\
parameter set 7 & 0.8 & 0.1 & 0.1 & 1 & 100 & 0.5 & $0.01 \mathrm{~K}$ & $7 \mathrm{~K}$ & $6 / \mathrm{K}$ \\
parameter set 8 & 0.2 & 0.05 & 0 & $0.1 / 3$ & 1 & 0.2 & $0.1 \mathrm{~K}$ & $2 \mathrm{~K}$ & $7 / \mathrm{K}$ \\
parameter set 9 & 0.2 & 0.07 & 0.1 & 3 & 100 & 0.6 & $0.1 \mathrm{~K}$ & $4 \mathrm{~K}$ & $6 / \mathrm{K}$ \\
\hline
\end{tabular}

Table 6: Model parameters and data

model with $\mu=0$ and $\gamma=0.35$ and with the other parameters as in parameter set 3 . To implement the RBF-FD method we further choose $M=400$ for the number of time steps. The exact prices are computed using the analytical formula in [30] for pricing European options. The reported absolute errors for the RBF-DQ and FD method are taken from [38, Table 4]. The results in Table 7 indicate that RBF-FD approximate option prices are in general more accurate than those computed by the RBF-DQ or the FD method. In particular for at-the-money and out-of the money option prices the RBF-FD method is much more accurate for this example.

\begin{tabular}{cccccc}
\hline & & RBF-DQ & FD & \multicolumn{2}{c}{ RBF-FD } \\
\hline$S$ & Exact price & Error & Error & Error & Value \\
\hline 0.25 & 0.000553 & $1.39 \mathrm{e}-4$ & $1.14 \mathrm{e}-4$ & $1.05 \mathrm{e}-6$ & 0.000551803 \\
0.50 & 0.021135 & $1.72 \mathrm{e}-6$ & $1.36 \mathrm{e}-6$ & $4.43 \mathrm{e}-6$ & 0.021130366 \\
0.75 & 0.120108 & $4.08 \mathrm{e}-6$ & $3.69 \mathrm{e}-5$ & $3.73 \mathrm{e}-6$ & 0.120104105 \\
1.00 & 0.301392 & $2.67 \mathrm{e}-5$ & $2.06 \mathrm{e}-4$ & $3.41 \mathrm{e}-6$ & 0.301388328 \\
1.25 & 0.525354 & $9.82 \mathrm{e}-5$ & $7.15 \mathrm{e}-4$ & $2.38 \mathrm{e}-8$ & 0.525353793 \\
1.50 & 0.765832 & $2.73 \mathrm{e}-4$ & $1.93 \mathrm{e}-3$ & $1.16 \mathrm{e}-7$ & 0.765831485 \\
1.75 & 1.012184 & $6.40 \mathrm{e}-4$ & $4.40 \mathrm{e}-3$ & $2.82 \mathrm{e}-7$ & 1.012184545 \\
\hline
\end{tabular}

Table 7: European call option prices under the Merton model with $\mu=0, \gamma=0.35$ and parameter set 3.

Further, we show how fast and efficient our method performs in comparison to other RBF methods in literature. In particular we compare our RBF-FD method to the RBF method in [8] and again the RBF-DQ method. We consider parameter set 4 . The corresponding exact European put price under the Merton model with $\mu=0$, $\gamma=0.5$ is 0.12299068 for $S=K=1$ and is computed using the analytical formula from [30]. The absolute errors at $S=K=1$ are reported in Table 8. For these computations, we fix $M=1080$ as the number of time steps but let the number of grid points $N$ vary. Table 6 of [38] reports CPU times of the order of seconds for the RBF method in [8] and for the RBF-DQ method. Comparing those to the CPU times for our RBF-FD method listed in Table 8 which are of the order of milliseconds, we may conclude that the RBF-FD method is efficient and fast.

\begin{tabular}{|c|c|c|c|c|c|}
\hline & RBF [8] & RBF-DQ & & RBF-FD & \\
\hline$N$ & Error & Error & Error & Value & CPU (s) \\
\hline \multicolumn{3}{|c|}{ Exact price } & \multicolumn{3}{|c|}{0.12299068} \\
\hline 80 & $8.30 \mathrm{e}-4$ & $6.22 \mathrm{e}-5$ & $3.7861 \mathrm{e}-5$ & 0.123039401 & 0.041 \\
\hline 160 & $2.03 \mathrm{e}-4$ & $1.51 \mathrm{e}-5$ & $5.7673 e-6$ & 0.123003307 & 0.087 \\
\hline 320 & $5.03 e-5$ & $3.61 \mathrm{e}-6$ & $2.4174 \mathrm{e}-6$ & 0.122993400 & 0.157 \\
\hline 640 & $1.26 e-5$ & $7.51 \mathrm{e}-7$ & $6.9075 \mathrm{e}-7$ & 0.122990413 & 0.677 \\
\hline
\end{tabular}

Table 8: European put option prices under the Merton model with $\mu=0, \gamma=0.5$ and parameter set 4 .

American put option prices under the Merton and the Kou model To further test our proposed RBF-FD method we compute the American put option prices under the Merton and the Kou model for the two parameter 
sets of Table 3. We report in Tables 9 and 10 the option prices for these options and their absolute error with respect to the reference prices from Table 5. In many papers in the literature the evaluation of option prices has been tested using these parameter sets. For the first parameter set we can compare our results with those in $[39,19,27,38,8,9,23,37]$ and for the second parameter set with those in [40, 37]. In particular we focus on [23, Tables 9 and 10] and on [27, Tables 5.1 and 5.3] since they use an RBF-FD method and an FD method, respectively, combined with an IMEX-scheme but both without first applying a coordinate transformation. We may conclude that our method leads to very accurate results using less time steps and spatial discretization points and in only a few milliseconds. Also in comparison to the methods in the other references our method is competitive since it needs less time steps and discretization points to get comparable or better accuracy. We can also easily compute the early exercise boundary using the approximate formula (see, e.g., [27])

$$
S_{p}(\tau)=\max \left\{S(x)>0 \mid \mathbf{U}^{k} \leq \mathbf{g}, x \in X, 0 \leq k \leq M\right\}
$$

where $\mathbf{U}^{k}$ is the numerical solution for the system (30)-(31) and $X$ is the set of interpolation points of [0,1]. In Figure 5 we provide the plot of the early exercise boundary for an American put option under the Merton model and the Kou model for parameter sets 1 and 2. The part below each curve represents the exercise or stopping region while the part above it is the holding or continuation region. We note that the exercise boundary decreases with increasing time to maturity.

\begin{tabular}{|c|c|c|c|c|c|c|c|c|}
\hline & & \multicolumn{2}{|c|}{$S=90$} & \multicolumn{2}{|c|}{$S=100$} & \multicolumn{2}{|c|}{$S=110$} & \multirow[b]{2}{*}{ CPU (s) } \\
\hline$N$ & $\bar{M}$ & Value & Error & Value & Error & Value & Error & \\
\hline \multicolumn{2}{|c|}{ Merton [13] } & 10.003822 & & 3.241251 & & 1.419803 & & \\
\hline 12 & 64 & 10.000713 & $3.1094 \mathrm{e}-3$ & 3.239611 & $1.6400 \mathrm{e}-3$ & 1.419435 & $3.6807 e-4$ & 0.005 \\
\hline 25 & 128 & 10.004088 & $2.6626 \mathrm{e}-4$ & 3.241030 & $2.2113 \mathrm{e}-4$ & 1.419741 & $6.1922 \mathrm{e}-5$ & 0.015 \\
\hline 51 & 256 & 10.003786 & $3.5994 \mathrm{e}-5$ & 3.241259 & $7.7127 \mathrm{e}-6$ & 1.419793 & $9.7920 \mathrm{e}-6$ & 0.121 \\
\hline \multicolumn{2}{|c|}{ Kou [45] } & 10.005071 & & 2.807879 & & 0.561876 & & \\
\hline 12 & 64 & 10.003149 & $1.9215 \mathrm{e}-3$ & 2.806248 & $1.6308 \mathrm{e}-3$ & 0.561578 & $2.9773 \mathrm{e}-4$ & 0.005 \\
\hline 25 & 128 & 10.005122 & $5.0730 \mathrm{e}-5$ & 2.807660 & $2.1858 \mathrm{e}-4$ & 0.561841 & $3.4580 \mathrm{e}-5$ & 0.013 \\
\hline 51 & 256 & 10.005127 & $5.6458 \mathrm{e}-5$ & 2.807866 & $1.2954 \mathrm{e}-5$ & 0.561861 & $1.5480 \mathrm{e}-5$ & 0.102 \\
\hline
\end{tabular}

Table 9: American put option prices under the Merton model and the Kou model for parameter set 1

\begin{tabular}{|c|c|c|c|c|c|c|c|c|}
\hline & & \multicolumn{2}{|c|}{$S=90$} & \multicolumn{2}{|c|}{$S=100$} & \multicolumn{2}{|c|}{$S=110$} & \\
\hline$N$ & $M$ & Value & Error & Value & Error & Value & Error & $\mathrm{CPU}(\mathrm{s})$ \\
\hline Merto & a [40] & 19.948906 & & 18.246332 & & 16.666925 & & \\
\hline 128 & 64 & 19.949471 & $5.6547 \mathrm{e}-04$ & 18.241667 & $4.6644 \mathrm{e}-03$ & 16.658594 & $8.3307 e-03$ & 0.005 \\
\hline 256 & 128 & 19.949048 & $1.4186 \mathrm{e}-04$ & 18.245175 & $1.1572 \mathrm{e}-03$ & 16.664860 & $2.0644 \mathrm{e}-03$ & 0.015 \\
\hline 512 & 256 & 19.948954 & $4.7847 \mathrm{e}-05$ & 18.246051 & $2.8081 \mathrm{e}-04$ & 16.666419 & $5.0570 \mathrm{e}-04$ & 0.120 \\
\hline Kou [4 & & 10.698208 & & 6.417275 & & 4.624099 & & \\
\hline 128 & 64 & 10.694761 & $3.4468 \mathrm{e}-03$ & 6.414181 & $3.0939 \mathrm{e}-03$ & 4.622385 & $1.7137 \mathrm{e}-03$ & 0.005 \\
\hline 256 & 128 & 10.697760 & $4.4816 \mathrm{e}-04$ & 6.416588 & $6.8678 \mathrm{e}-04$ & 4.623680 & $4.1880 \mathrm{e}-04$ & 0.014 \\
\hline 512 & 256 & 10.698363 & $1.5476 \mathrm{e}-04$ & 6.417157 & $1.1766 \mathrm{e}-04$ & 4.624006 & $9.3047 \mathrm{e}-05$ & 0.105 \\
\hline
\end{tabular}

Table 10: American put option prices under the Merton model and the Kou model for parameter set 2

Short and long maturity combined with low and high volatility In [8] special focus is put on the accuracy in option pricing for small maturities. Hereto, an adaptive RBF method was proposed. We compute by our RBFFD method the European put option prices for the Merton model with the first parameter set and let $T$ vary, i.e. $T=1 \mathrm{e}-6,0.25,1$. Further, we consider two cases for the volatility $\sigma=0.15$ and $\sigma=1$. We report the relative error to be able to compare with the reported relative errors in [8, Table 5]. We conclude from Table 11 that our RBF-FD method remains very accurate even for very short maturities combined with either low $(\sigma=0.15)$ or high $(\sigma=1)$ volatility when using only $N=M=512$ discretization points in space and time. Comparing to [8, 

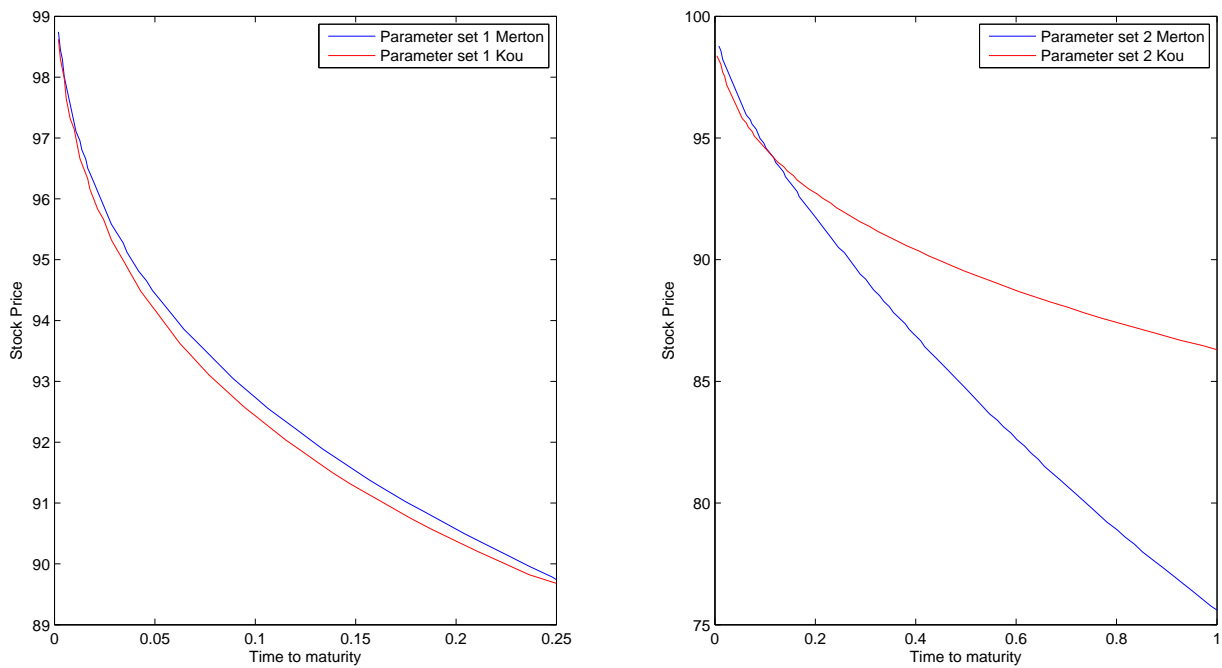

Figure 5: Early exercise boundary for an American put option under the Merton and the Kou models for parameter sets 1 and 2.

Table 5] for $\sigma=0.15$ we observe that our RBF-FD method is more accurate than the global method in [8] since with a comparable number of spatial discretization points we get smaller errors or in one case a comparable error.

\begin{tabular}{cccccccc}
\hline \multicolumn{7}{c}{$\sigma=0.15$} & \multicolumn{2}{c}{$\sigma=1$} \\
\hline$T$ & $S$ & Value & Rel. Error & Exact price & Value & Rel. Error & Exact price \\
\hline \multirow{3}{*}{$1 \mathrm{e}-06$} & 99.9 & 0.099995 & $3.169360 \mathrm{e}-8$ & $9.999504 \mathrm{e}-02$ & 0.108330 & $1.2406 \mathrm{e}-04$ & 0.108316 \\
& 100 & $5.742706 \mathrm{e}-3$ & $4.039229 \mathrm{e}-2$ & $5.984431 \mathrm{e}-03$ & 0.039840 & $1.3741 \mathrm{e}-03$ & 0.039895 \\
& 100.1 & $4.503670 \mathrm{e}-6$ & $1.873367 \mathrm{e}-1$ & $5.541865 \mathrm{e}-06$ & 0.008354 & $8.0340 \mathrm{e}-04$ & 0.008348 \\
\hline & 90 & 9.285433 & $1.5853 \mathrm{e}-06$ & 9.285418 & 23.655246 & $9.8890 \mathrm{e}-07$ & 23.655223 \\
& 99.9 & 3.184844 & $2.2213 \mathrm{e}-06$ & 3.184837 & 19.437222 & $1.0726 \mathrm{e}-06$ & 19.437202 \\
0.25 & 100 & 3.149033 & $2.2659 \mathrm{e}-06$ & 3.149026 & 19.398611 & $1.0656 \mathrm{e}-06$ & 19.398590 \\
& 100.1 & 3.113710 & $2.3119 \mathrm{e}-06$ & 3.113703 & 19.360075 & $1.0584 \mathrm{e}-06$ & 19.360055 \\
& 110 & 1.401192 & $4.3717 \mathrm{e}-06$ & 1.401186 & 15.900450 & $1.1337 \mathrm{e}-06$ & 15.900468 \\
\hline & 90 & 10.30366 & $2.8981 \mathrm{e}-05$ & 10.303963 & 38.857797 & $5.4859 \mathrm{e}-05$ & 38.859928 \\
& 95 & 8.189224 & $2.5239 \mathrm{e}-05$ & 8.189431 & 37.303492 & $5.1461 \mathrm{e}-05$ & 37.305412 \\
1 & 100 & 6.684271 & $2.5524 \mathrm{e}-05$ & 6.684441 & 35.839436 & $4.9390 \mathrm{e}-05$ & 35.841206 \\
& 105 & 5.654291 & $1.8398 \mathrm{e}-05$ & 5.654395 & 34.458796 & $4.7071 \mathrm{e}-05$ & 34.460419 \\
& 110 & 4.961362 & $1.7882 \mathrm{e}-05$ & 4.961450 & 33.155272 & $4.6061 \mathrm{e}-05$ & 33.156799 \\
\hline
\end{tabular}

Table 11: European put option prices under the Merton model with parameter set 1 and varying $T$ and $\sigma$. Other parameters are $N=512, M=512, S_{\min }=0.01 K$, and $S_{\max }=2 K, 4 K, 10 K$ for $T=1 \mathrm{e}-06,0.25,1$, respectively and $\zeta=1 / K, 7 / K$ for $\sigma=0.15,1$, respectively.

Also [38] tested their RBF method for a short maturity of $T=0.1$ combined with a low $(\sigma=0.1)$ and a high $(\sigma=1)$ volatility. The prices of a European put option under the Merton model with $\mu=0$ and $\gamma=0.35$ and parameter set 5 from Table 6 are computed for 1950 equally spaced stock prices in the interval $[K / 3,5 K / 3]$ and the corresponding RMS error is reported. Note that hereto first RBF-FD approximations are computed using $N=128,256$ and 512 spatial grid points combined with $M=64,128$ and 256 time steps respectively. Next, the option prices at these 1950 equally spaced stock prices are obtained by interpolating the solution. Comparing these RMS errors in Table 12 obtained by our RBF-FD method with those in [38, Table 7], we conclude that our method leads to a higher accuracy with less spatial and time discretization points. To get an error of order $1 \mathrm{e}-07$ $2560(\sigma=0.1)$ and $1280(\sigma=1)$ nodal grid points are needed in [38], while we get this order of accuracy using 
$N+1=513$ grid points.

\begin{tabular}{cccc}
\hline & & $\sigma=0.1$ & $\sigma=1$ \\
\hline$N$ & $M$ & RMS error & RMS error \\
\hline 128 & 64 & $6.1971 \mathrm{e}-06$ & $1.0256 \mathrm{e}-05$ \\
256 & 128 & $1.2249 \mathrm{e}-06$ & $2.0468 \mathrm{e}-06$ \\
512 & 256 & $2.7987 \mathrm{e}-07$ & $6.2346 \mathrm{e}-07$ \\
\hline
\end{tabular}

Table 12: European put option prices under the Merton model with $\mu=0, \gamma=0.35$ and parameter set 5.

In addition, we study the convergence rate in time and space for a European put option under a Merton model with $\mu=0, \gamma=0.35$, combined with parameter set 5 and for low $(\sigma=0.1)$ and high $(\sigma=1)$ volatility. Figure 6 confirms that the proposed method is also in these cases second order in time and space.
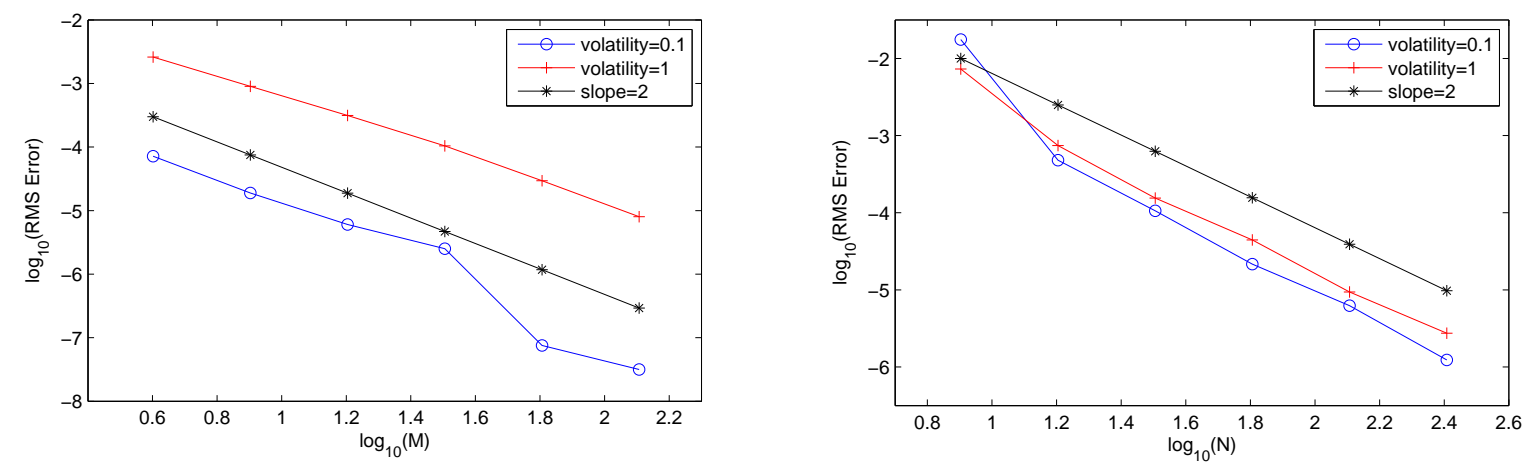

Figure 6: European put option under the Merton model with $\mu=0, \gamma=0.35$ and parameter set 5. Left: Error convergence in time using 2048 nodal points. Right: Error convergence in space using 512 time discretization steps.

We now test a long maturity in combination with low and high volatilities. For European put options we have already some results for $T=3$ in Table 7 and for $T=1$ in Table 11 . In Table 13 we consider again a long maturity, $T=3$, for a European put option under the Merton model with $\mu=0$ and $\gamma=0.35$ but with parameter set 6 in order to compare to [38, Table 9]. Also in this test our RBF-FD method proves to be accurate and fast. Our method leads to an error of order 1e-06 with $N=512$ for the spatial discretization and in a few milliseconds while in [38] 640 spatial points give an error of order 1e-05 and they report 9.8 seconds to get to this precision.

\begin{tabular}{lcccc}
\hline \multicolumn{5}{c}{$S=100$} \\
\hline$N$ & $M$ & Value & Error & CPU (s) \\
\hline \multicolumn{2}{l}{ Exact price } & 9.8233158 & & \\
\hline 128 & 256 & 9.8229636 & $3.5221 \mathrm{e}-04$ & 0.014 \\
256 & 512 & 9.8232739 & $4.1847 \mathrm{e}-05$ & 0.080 \\
512 & 1024 & 9.8233228 & $7.0328 \mathrm{e}-06$ & 0.355 \\
\hline
\end{tabular}

Table 13: European put option prices under the Merton model with $\mu=0, \gamma=0.35$ and parameter set 6 .

For an American put option with a long maturity $T=1$ and a high volatility $\sigma=0.8$ we can compare our results in Table 14 to those in [38, Table 15]. The benchmark option price is taken from [38, Table 15] and is evaluated using the proposed method by [28]. Also for the American case our RBF-FD method is very fast and accurate since we need only $N+1=513$ spatial discretization nodes to get an error of order 1e-05 in some milliseconds while in [38] they report for $N=640$ an error of 1e-04 in 17.11 seconds. In addition we provide in Figure 7 the plot of the early exercise boundary for an American put option under the Merton model for parameter sets 7 and 9. Under each curve we find the exercise or stopping region while the part above it is the continuation or holding region. 


\begin{tabular}{ccccc}
\hline \multicolumn{5}{c}{$S=100$} \\
\hline$N$ & $M$ & Value & Error & CPU (s) \\
\hline \multicolumn{6}{c}{ Benchmark } & 29.832970 & & \\
\hline 128 & 256 & 29.8359417 & $2.9717 \mathrm{e}-03$ & 0.011 \\
256 & 512 & 29.8334683 & $4.9829 \mathrm{e}-04$ & 0.051 \\
512 & 1024 & 29.8329819 & $1.1932 \mathrm{e}-05$ & 0.317 \\
\hline
\end{tabular}

Table 14: American put option prices under the Merton model with $\mu=0, \gamma=0.3$ and parameter set 7.
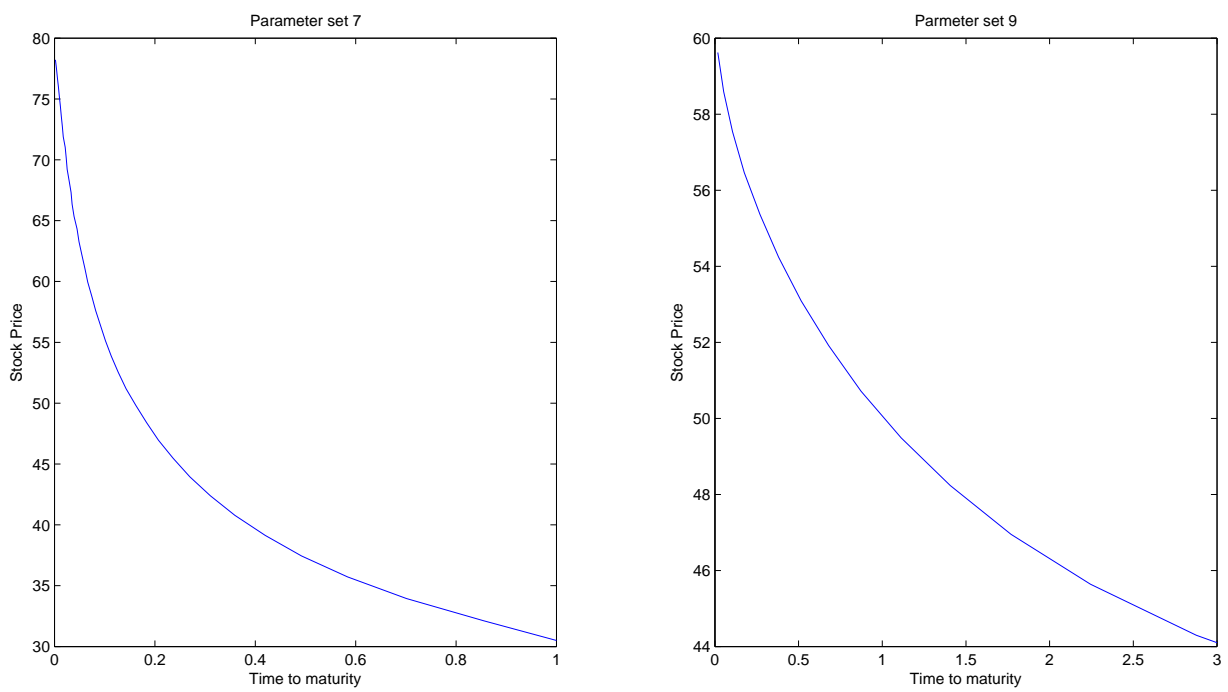

Figure 7: Early exercise boundary for American put option under the Merton model for parameter sets 7 and 9.

\subsection{Greeks}

In this section our aim is to evaluate the efficiency of the RBF-FD method against other existing methods in the literature for computing the Greeks $\Delta=\frac{\partial V}{\partial S}$ and $\Gamma=\frac{\partial^{2} V}{\partial S^{2}}$ introduced in section 6.

We start by considering a European put option with short maturity $T=0.1$ under the Merton model with $\mu=0, \gamma=0.35$ and parameter set 8 . These parameters have been used for evaluating $\Delta$ and $\Gamma$ in [38] using the RBF-DQ method. As in [38] we compute the RMS errors and CPU times, see Table 15. For computing these RMS errors, we apply the RBF-FD method and use the values of the hedging parameters $\Delta$ and $\Gamma$ in 1950 equally spaced stock prices in the range of $[K / 3,5 K / 3]$. The exact values of $\Delta$ and $\Gamma$ in the expression of the RMS error for the different stock prices have been computed by using the closed form solution presented in [30]. Now, comparing our results with those in [38, Table 18], we may conclude that the RBF-FD method is more accurate than the RBF-DQ method also for computing the Greeks. For achieving a same level of accuracy, the RBF-FD method needs to solve a linear system of equations of smaller size than in the RBF-DQ method. Moreover the coefficient matrix in the RBF-FD method is tridiagonal, implying that the system can be solved very fast.

\begin{tabular}{ccccc}
\hline \multicolumn{5}{c}{ RMS error } \\
\hline$N$ & $M$ & $\Delta$ & $\Gamma$ & CPU (s) \\
\hline 256 & 128 & $4.8831 \mathrm{e}-05$ & $2.3144 \mathrm{e}-04$ & 0.010 \\
512 & 256 & $1.2047 \mathrm{e}-05$ & $7.2280 \mathrm{e}-05$ & 0.152 \\
1024 & 512 & $3.0479 \mathrm{e}-06$ & $1.7567 \mathrm{e}-05$ & 0.513 \\
2048 & 1024 & $8.0647 \mathrm{e}-07$ & $7.2174 \mathrm{e}-06$ & 4.327 \\
\hline
\end{tabular}

Table 15: Delta and Gamma of European put option price under the Merton model with $\mu=0, \gamma=0.35$ and parameter set 8 for a short maturity $T=0.1$. 
To further investigate the efficiency of the RBF-FD method, we consider again a European put option under the Merton model with $\mu=0, \gamma=0.35$ and parameter set 8 but with a long maturity $T=3$. The absolute errors and the RMS errors when computing the Greeks for this put option for varying values of $S$ using the RBF-FD method with $N=1024$ and $M=1024$ are reported in Table 16 . We conclude that also in this case the RBF-FD method is accurate.

\begin{tabular}{|c|c|c|c|c|c|c|}
\hline$S$ & $\Delta$ & Exact value & Error & $\Gamma$ & Exact value & Error \\
\hline 80 & -0.49307119 & -0.493067335 & $3.8569 \mathrm{e}-06$ & 0.01191453 & 0.011914579 & $3.4749 \mathrm{e}-08$ \\
\hline 85 & -0.43527507 & -0.435271821 & $3.2536 \mathrm{e}-06$ & 0.01117259 & 0.011172598 & $2.8725 \mathrm{e}-08$ \\
\hline 90 & -0.38158922 & -0.381586517 & $2.7046 \mathrm{e}-06$ & 0.01028331 & 0.010283331 & $1.8816 \mathrm{e}-08$ \\
\hline 95 & -0.33256734 & -0.332565092 & $2.2489 \mathrm{e}-06$ & 0.00931775 & 0.009317751 & $7.7364 \mathrm{e}-09$ \\
\hline 100 & -0.28844229 & -0.288440390 & $1.9022 \mathrm{e}-06$ & 0.00833293 & 0.008332941 & $3.6005 e-08$ \\
\hline 105 & -0.24919839 & -0.249196723 & $1.6648 \mathrm{e}-06$ & 0.00737123 & 0.007371226 & $4.3037 \mathrm{e}-08$ \\
\hline 110 & -0.21464169 & -0.214640165 & $1.5282 \mathrm{e}-06$ & 0.00646189 & 0.006461887 & $1.2793 \mathrm{e}-08$ \\
\hline 115 & -0.18446145 & -0.184459970 & $1.4805 \mathrm{e}-06$ & 0.00562287 & 0.005622860 & $2.1484 \mathrm{e}-08$ \\
\hline 120 & -0.15827982 & -0.158278311 & $1.5095 \mathrm{e}-06$ & 0.00486340 & 0.004863397 & $2.9209 \mathrm{e}-08$ \\
\hline \multicolumn{3}{|c|}{ RMS error at the above 9 points } & $2.3812 \mathrm{e}-06$ & & & $2.8059 \mathrm{e}-08$ \\
\hline
\end{tabular}

Table 16: Delta and Gamma of European put option price under the Merton model with $\mu=0, \gamma=0.35$ and parameter set 8 for a long maturity $T=3$.

Since there is no closed form formula for American option prices and their Greeks we test the RBF-FD method for the computation of the Greeks of an American put option under the Merton model with a parameter set as in [38]. Thus we put $\mu=0, \gamma=0.35$ and consider the parameter set 9 which is a case of a long maturity $T=3$. Numerical approximations for the Greeks $\Delta$ and $\Gamma$ of this American option obtained by the RBF-FD method using different numbers of time and space steps and different stock prices are reported in Table 17. Comparing to [38, Table 20] we conclude that the results for the Greeks are in line with each other.

\begin{tabular}{ccccc}
\hline \multicolumn{3}{c}{$\Delta$} & \multicolumn{2}{c}{$\Gamma$} \\
\hline$S$ & $N=512, M=256$ & $N=1024, M=512$ & $N=512, M=256$ & $N=1024, M=512$ \\
\hline 80 & -0.50461524776 & -0.50459969084 & 0.00798701879 & 0.00798658642 \\
85 & -0.46612448531 & -0.46611327509 & 0.00742345331 & 0.00742318607 \\
90 & -0.43026138170 & -0.43025105856 & 0.00693080288 & 0.00693058531 \\
95 & -0.39674621849 & -0.39673689636 & 0.00648062636 & 0.00648046681 \\
100 & -0.36540995868 & -0.36540193399 & 0.00611455180 & 0.00607728393 \\
105 & -0.33614380923 & -0.33613591696 & 0.00565209011 & 0.00565202013 \\
110 & -0.30886416789 & -0.30885694770 & 0.00526193679 & 0.00526184852 \\
115 & -0.28350160200 & -0.28349409671 & 0.00488561810 & 0.00488553516 \\
120 & -0.25998251566 & -0.25997703555 & 0.00452359882 & 0.00452353538 \\
\hline
\end{tabular}

Table 17: Delta and Gamma of American put option price under the Merton model with $\mu=0, \gamma=0.35$ and parameter set 9 .

For the parameter set 1 combined with the jump distribution parameters from Table 2, we plotted in Figures 8 and 9 the Delta and Gamma functions against the exact values for European put option prices under the Kou and Merton model. Also, for the American put option prices under the Kou and Merton model with the same parameters as in the European case, we depicted the option price and its Delta and Gamma functions in Figures 10 and 11 .

All plots are smooth with no oscillations at or around the strike. This shows that the RBF-FD technique combined with a stable time discretization is accurate for approximating the option Greeks Delta and Gamma.

\section{Conclusions}

We presented an efficient numerical method that is second order in time and space to evaluate European and American option prices based on a combination of the RBF-FD method and a three level IMEX scheme that treats 

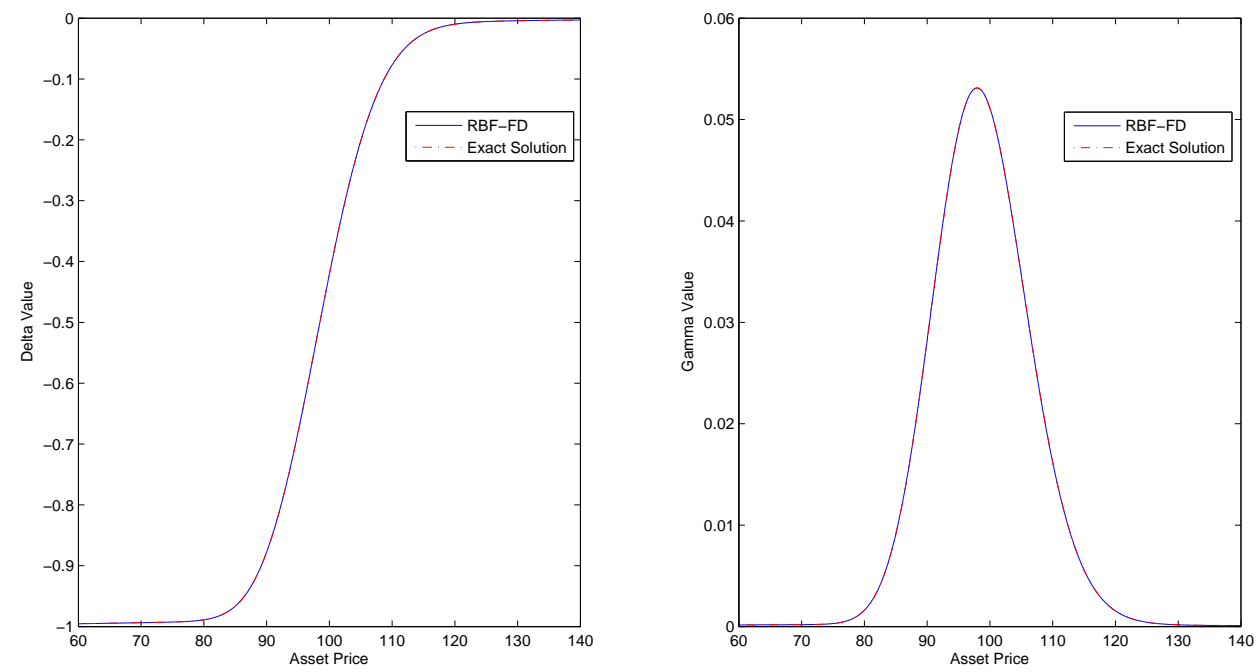

Figure 8: Delta and Gamma of European put option price under the Kou model with jump distribution parameters from Table 2 and with parameter set 1.
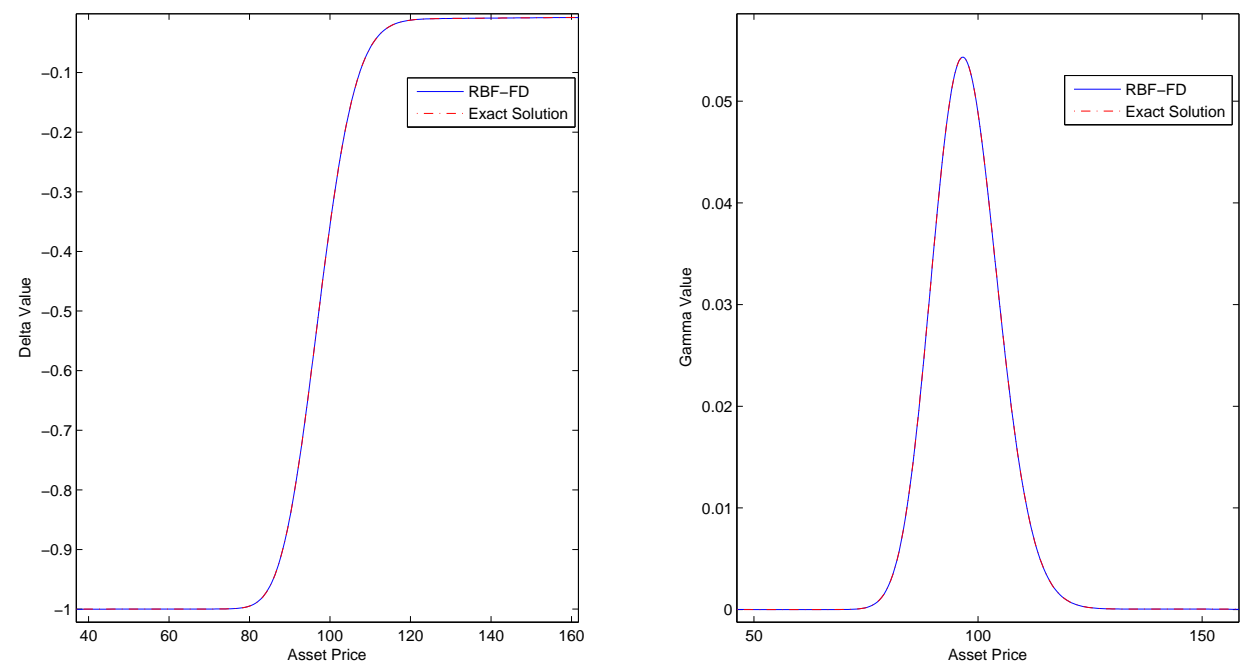

Figure 9: Delta and Gamma of European put option price under the Merton model with with jump distribution parameters from Table 2 and parameter set 1. 

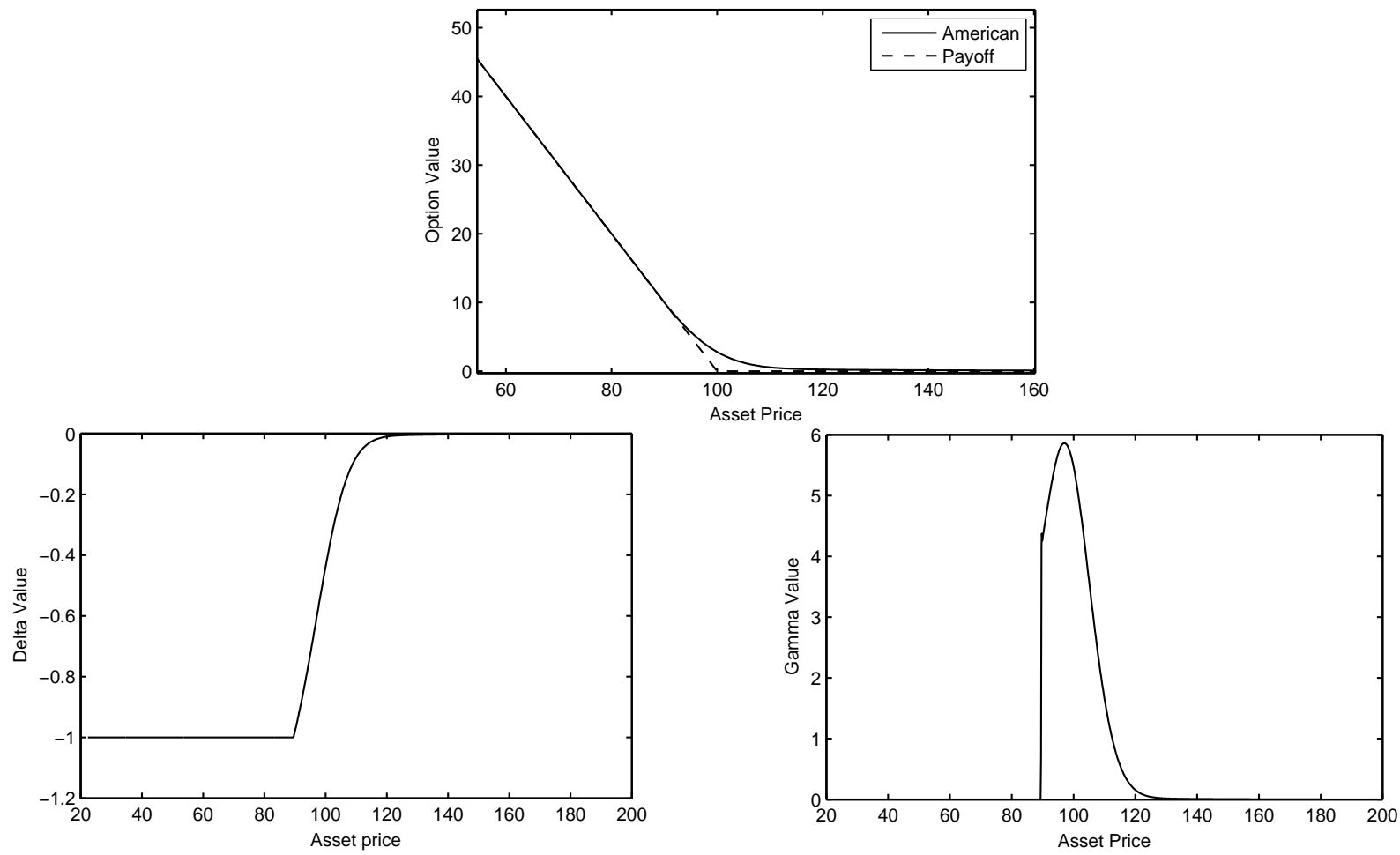

Figure 10: American put option price and its Delta and Gamma under the Kou model with jump distribution parameters from Table 2 and with parameter set 1.
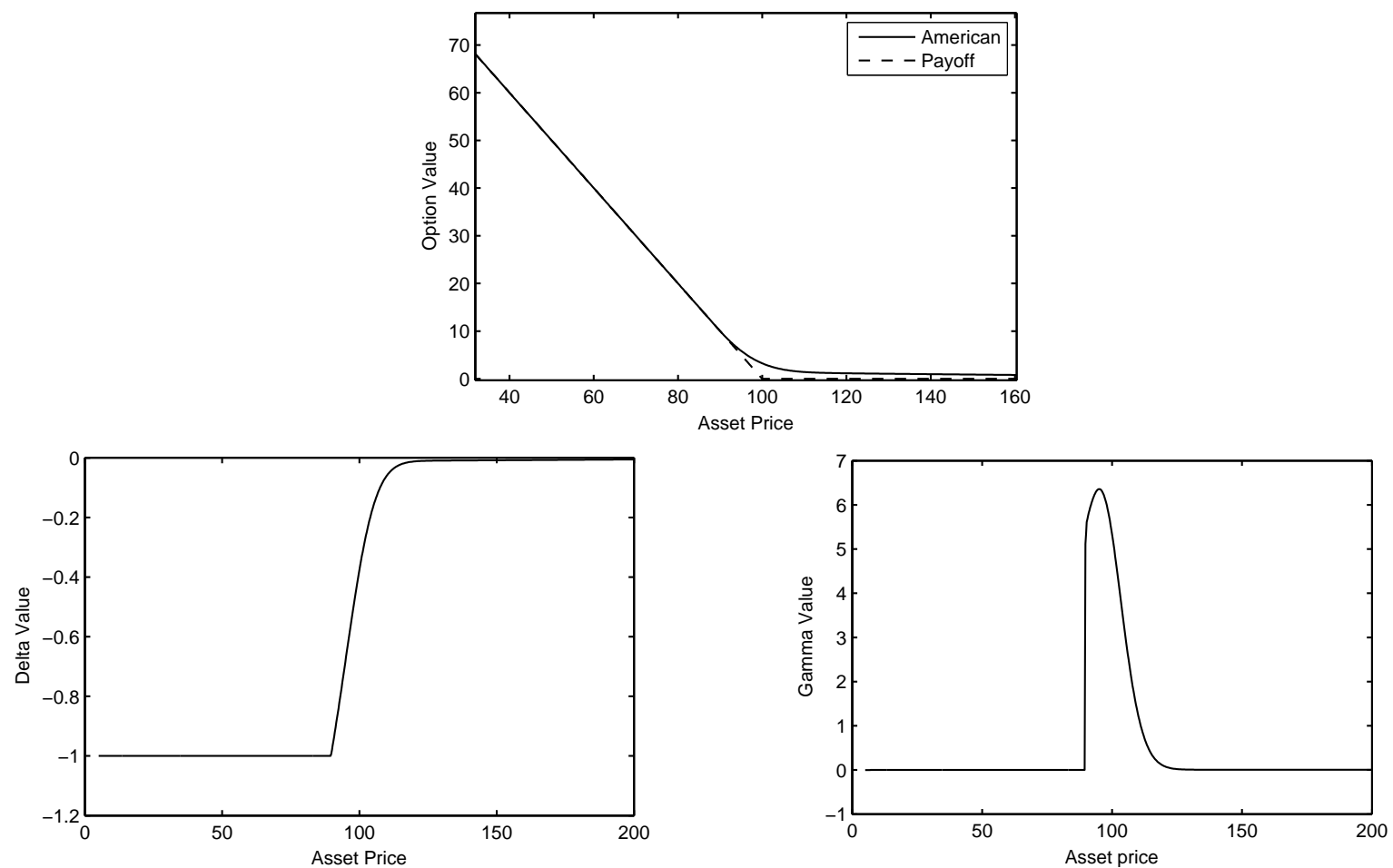

Figure 11: American put option price and its Delta and Gamma under the Merton model with with jump distribution parameters from Table 2 and parameter set 1. 
the non-local integral term explicitly. An important difference to other schemes in the literature is that we first transformed the PIDE by a coordinate stretching coordinate transformation so that the computation of the prices is focused on the regions of interest. We proved for the European option case that our scheme is stable and second order convergent in time and space. For American options this was confirmed by the numerical experiments. We compared several cases including long and short maturities, low and high volatility. We also tested the performance to approximate the hedging Greeks Delta and Gamma. We observed that the proposed method is very accurate and efficient in all those cases.

\section{References}

[1] L. Andersen and J. Andreasen. Jump-diffusion processes: Volatility smile fitting and numerical methods for option pricing. Review of Derivatives Research, 4(3):231-262, 2000.

[2] U.M. Ascher, S.J. Ruuth, and B.T.R. Wetton. Implicit-explicit methods for time-dependent partial differential equations. SIAM Journal on Numerical Analysis, 32(3):797-823, 1995.

[3] L.V. Ballestra and G. Pacelli. Pricing European and American options with two stochastic factors: A highly efficient radial basis function approach. Journal of Economic Dynamics and Control, 37(6):1142-1167, 2013.

[4] L.V. Ballestra and C. Sgarra. The evaluation of American options in a stochastic volatility model with jumps: An efficient finite element approach. Computers \& Mathematics with Applications, 60(6):1571-1590, 2010.

[5] V. Bayona, M. Moscoso, M. Carretero, and M. Kindelan. RBF-FD formulas and convergence properties. Journal of Computational Physics, 229(22):8281-8295, 2010.

[6] M. Briani, R. Natalini, and G. Russo. Implicit-explicit numerical schemes for jump-diffusion processes. Calcolo, 44(1):33-57, 2007.

[7] R.T.L. Chan. Adaptive radial basis function methods for pricing options under jump-diffusion models. Computational Economics, 47(4):623-643, Apr 2016.

[8] R.T.L. Chan and S. Hubbert. Options pricing under the one-dimensional jump-diffusion model using the radial basis function interpolation scheme. Review of Derivatives Research, 17(2):161-189, 2014.

[9] C.C. Christara and N.C.-H. Leung. Option pricing in jump diffusion models with quadratic spline collocation. Applied Mathematics and Computation, 279:28-42, 2016.

[10] N. Clarke and K. Parrott. Multigrid for American option pricing with stochastic volatility. Applied Mathematical Finance, 6(3):177-195, 1999.

[11] R. Cont and E. Voltchkova. A finite difference scheme for option pricing in jump diffusion and exponential Lévy models. SIAM Journal on Numerical Analysis, 43(4):1596-1626, 2005.

[12] Y. d'Halluin, P.A. Forsyth, and G. Labahn. A penalty method for American options with jump diffusion processes. Numerische Mathematik, 97(2):321-352, 2004.

[13] Y. d'Halluin, P.A. Forsyth, and K.R. Vetzal. Robust numerical methods for contingent claims under jump diffusion processes. IMA Journal of Numerical Analysis, 25(1):87-112, 2005.

[14] T.A. Driscoll and A.R.H. Heryudono. Adaptive residual subsampling methods for radial basis function interpolation and collocation problems. Computers \& Mathematics with Applications, 53(6):927-939, 2007.

[15] G.E. Fasshauer, A.Q.M. Khaliq, and D.A. Voss. Using meshfree approximation for multi asset American options. Journal of the Chinese Institute of Engineers, 27(4):563-571, 2004.

[16] B. Fornberg and N. Flyer. A Primer on Radial Basis Functions with Applications to the Geosciences. SIAM, Philadelphia, 2015.

[17] B. Fornberg and N. Flyer. Solving PDEs with radial basis functions. Acta Numerica, 24:215-258, 2015. 
[18] X.-T. Gan, J.-F. Yin, and Y.-X. Guo. Finite volume method for pricing European and American options under jump-diffusion models. East Asian Journal on Applied Mathematics, 7(2):227-247, 2017.

[19] A. Golbabai, D. Ahmadian, and M. Milev. Radial basis functions with application to finance: American put option under jump diffusion. Mathematical and Computer Modelling, 55(3):1354-1362, 2012.

[20] A.D. Holmes, H. Yang, and S. Zhang. A front-fixing finite element method for the valuation of American options with regime switching. International Journal of Computer Mathematics, 89(9):1094-1111, 2012.

[21] S. Ikonen and J. Toivanen. Operator splitting methods for American option pricing. Applied Mathematics Letters, 17(7):809-814, 2004.

[22] M.K. Kadalbajoo, A. Kumar, and L.P. Tripathi. Application of the local radial basis function-based finite difference method for pricing American options. International Journal of Computer Mathematics, 92(8):1608$1624,2015$.

[23] M.K. Kadalbajoo, A. Kumar, and L.P. Tripathi. A radial basis function based implicit-explicit method for option pricing under jump-diffusion models. Applied Numerical Mathematics, 110:159-173, 2016.

[24] M.K. Kadalbajoo, L.P. Tripathi, and A. Kumar. Second order accurate IMEX methods for option pricing under Merton and Kou jump-diffusion models. Journal of Scientific Computing, 65(3):979-1024, 2015.

[25] S.G. Kou. A jump-diffusion model for option pricing. Management Science, 48(8):1086-1101, 2002.

[26] Y. Kwon and Y. Lee. A second-order finite difference method for option pricing under jump-diffusion models. SIAM Journal on Numerical Analysis, 49(6):2598-2617, 2011.

[27] Y. Kwon and Y. Lee. A second-order tridiagonal method for American options under jump-diffusion models. SIAM Journal on Scientific Computing, 33(4):1860-1872, 2011.

[28] R. Lord, F. Fang, F. Bervoets, and C.W. Oosterlee. A fast and accurate FFT-based method for pricing earlyexercise options under Lévy processes. SIAM Journal on Scientific Computing, 30(4):1678-1705, 2008.

[29] S. Memon. Finite element method for American option pricing: a penalty method. International Journal of Numerical Analysis and Modeling, Series B, 3(3):345-370, 2012.

[30] R. Merton. Option pricing when underlying stock returns are discontinuous. Journal of Financial Economics, 3(1-2):125-144, 1976.

[31] R. Mollapourasl, A. Fereshtian, H. Li, and X. Lu. RBF-PU method for pricing options under the jumpdiffusion model with local volatility. Journal of Computational and Applied Mathematics, 337:98-118, 2018.

[32] R. Mollapourasl, A. Fereshtian, and M. Vanmaele. Radial basis functions with partition of unity method for American options with stochastic volatility. Computational Economics, Sep 2017.

[33] E. Ngounda, K.C. Patidar, and E. Pindza. Contour integral method for European options with jumps. Communications in Nonlinear Science and Numerical Simulation, 18(3):478-492, 2013.

[34] C.W. Oosterlee, C.C.W. Leentvaar, and X. Huang. Accurate American option pricing by grid stretching and high order finite differences. Technical report, Delft Institute of Applied Mathematics (DIAM), Delft University of Technology, 2005.

[35] E. Pindza, K.C. Patidar, and E. Ngounda. Robust spectral method for numerical valuation of European options under Merton's jump-diffusion model. Numerical Methods for Partial Differential Equations, 30(4):1169-1188, 2014.

[36] R.J. Plemmons. M-matrix characterizations.I-nonsingular M-matrices. Linear Algebra and its Applications, 18(2):175 -188, 1977.

[37] J.A. Rad and K. Parand. Pricing American options under jump-diffusion models using local weak form meshless techniques. International Journal of Computer Mathematics, 94(8):1694-1718, 2017. 
[38] A.A.E.F. Saib, D.Y. Tangman, and M. Bhuruth. A new radial basis functions method for pricing American options under Merton's jump-diffusion model. International Journal of Computer Mathematics, 89(9):11641185, 2012.

[39] S. Salmi and J. Toivanen. An iterative method for pricing American options under jump-diffusion models. Applied Numerical Mathematics, 61(7):821-831, 2011.

[40] S. Salmi and J. Toivanen. Comparison and survey of finite difference methods for pricing American options under finite activity jump-diffusion models. International Journal of Computer Mathematics, 89(9):11121134, 2012.

[41] S. Salmi and J. Toivanen. IMEX schemes for pricing options under jump-diffusion models. Applied Numerical Mathematics, 84:33-45, 2014.

[42] R. Seydel. Tools for Computational Finance. Springer, Berlin, Heidelberg, 4th edition, 2009.

[43] C. Shu, H. Ding, and K.S. Yeo. Local radial basis function-based differential quadrature method and its application to solve two-dimensional incompressible Navier-Stokes equations. Computer Methods in Applied Mechanics and Engineering, 192(78):941-954, 2003.

[44] J.C. Strikwerda. Finite difference Difference Schemes and Partial Differential Equations. SIAM, Philadelphia, second edition, 2004.

[45] J. Toivanen. Numerical valuation of European and American options under Kou's jump-diffusion model. SIAM Journal on Scientific Computing, 30(4):1949-1970, 2008.

[46] A.I. Tolstykh and D.A. Shirobokov. On using radial basis functions in a "finite difference mode" with applications to elasticity problems. Computational Mechanics, 33(1):68-79, 2003.

[47] G.B. Wright. Radial basis function interpolation: numerical and analytical developments. Ph.D. thesis, University of Colorado, Boulder, 2003.

[48] K. Zhang and S. Wang. Pricing options under jump diffusion processes with fitted finite volume method. Applied Mathematics and Computation, 201(12):398-413, 2008.

[49] R. Zhang, H. Song, and N. Luan. Weak Galerkin finite element method for valuation of American options. Frontiers of Mathematics in China, 9(2):455-476, Apr 2014. 